https://doi.org/10.15407/ujpe65.4.342

V.O. GUBANOV, A.P. NAUMENKO, M.M. BILYI, I.S. DOTSENKO, M.M. SABOV, M.S. IAKHNENKO, L.A. BULAVIN

Taras Shevchenko National University of Kyiv, Faculty of Physics (64/13, Volodymyrs'ka Str., Kyiv 01601, Ukraine)

\title{
ENERGY SPECTRA OF ELECTRON EXCITATIONS IN GRAPHITE AND GRAPHENE AND THEIR DISPERSION MAKING ALLOWANCE FOR THE ELECTRON SPIN AND THE TIME-REVERSAL SYMMETRY
}

\begin{abstract}
The dispersion dependences of electron excitations in crystalline graphite and single-layer graphene have been studied taking the electron spin into consideration. The correlations of the energy spectra of electron excitations and, for the first time, the compatibility conditions for two-valued irreducible projective representations characterizing the symmetry of spinor excitations in the indicated structures are determined, as well as the distributions of spinor quantum states over the projective classes and irreducible projective representations for all highsymmetry points in the corresponding Brillouin zones. With the help of theoretical symmetrygroup methods for the spatial symmetry groups of crystalline graphite and single-layer graphene (in particular, the splitting of $\pi$-bands at the Dirac points), the spin-dependent splittings in their electron energy spectra are found. The splitting magnitude can be considerable, e.g., for dichalcogenides of transition metals belonging to the same spatial symmetry group. But it is found to be small for crystalline graphite and single-layer graphene because of a low spin-orbit interaction energy for carbon atoms and, as a consequence, carbon structures.
\end{abstract}

Keywords: crystalline graphite, single-layer graphene, spinor representations, factorsystems, dispersion of electron excitations, projective classes, two-valued irreducible projective representations.

\section{Introduction}

In work [1], a theoretical symmetry-group description was presented for the dispersion of vibrational and electron excitations in crystalline graphite. The analysis was carried out on the basis of projective classes of representations following from the spatial symmetry of crystalline graphite structure and determined at various points of the corresponding Brillouin zone. For high-symmetry points in the Brillouin zone of crystalline graphite, irreducible projective representations were constructed according to which the wave functions of elementary excitations in this substance are transformed. In work [1], correlations between phonon and electron excitations in graphite and single-layer graphene were also demonstrated. For both structures, only $\pi$-bands - i.e. the

(C) V.O. GUBANOV, A.P. NAUMENKO, M.M. BILYI, I.S. DOTSENKO, M.M. SABOV, M.S. IAKHNENKO, L.A. BULAVIN, 2020 electron bands of $\pi$-orbitals producing $\pi$-electrons and $\pi$-holes, whose wave functions are orthogonal to the functions of $\sigma$-zones of $s p^{2}$-hybridized $\sigma$-orbitals were considered as electron ones. In so doing, we did not consider the spin-orbit interaction for electron states, because it is insignificant for $\pi$-bands in carbon structures [2].

The symmetry of the crystal lattice of Bernal graphite [3] is described by the spatial group $P 6_{3} / m m c\left(D_{6 h}^{4}\right)$, which is also the spatial symmetry group of the crystal lattices of hexagonal boron nitride $(\mathrm{BN})$ and hexagonal polytypes $2 \mathrm{H}_{a}$ and $2 \mathrm{H}_{c}$ of the dichalcogenides of transition metals $\left(\mathrm{MoS}_{2}\right.$, $\mathrm{MoSe}_{2}, \mathrm{WS}_{2}, \mathrm{WSe}_{2}, \mathrm{TeS}_{2}$, and $\mathrm{TeSe}_{2}$ ). Therefore, it was important for us to determine the qualitative character of the influence of an electron spin on the structure of $\pi$-bands in graphite and other compounds, whose crystal lattice is described by the spatial symmetry group $P 6_{3} / m m c\left(D_{6 h}^{4}\right)$. Another issue, also important for us, was to consider the influence of

ISSN 2071-0194. Ukr. J. Phys. 2020. Vol. 65, No. 4 

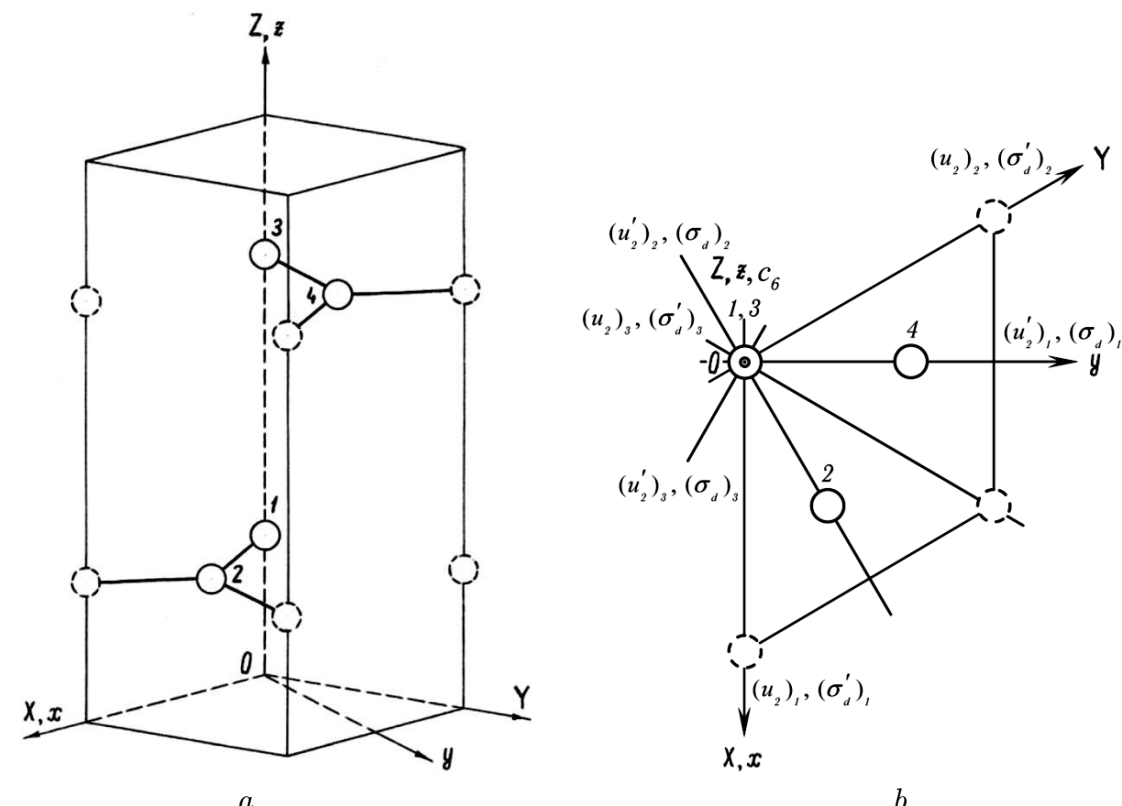

Fig. 1. Structure of a standard unit cell of graphite crystals $\gamma-C(a)$ and arrangement and orientation of the elements of the point symmetry group $6 / \mathrm{mmm}\left(D_{6 h}\right)(b)$. Circles indicate the positions of carbon atoms (reproduced from work [1])

the time-reversal symmetry on elementary excitations in lattices with the indicated spatial symmetry.

\section{Standard Unit Cells, Brillouin Zones, and Basic Symmetry Elements of Graphite and Single-Layer Graphene}

In Fig. 1, $a$, a standard unit cell of the crystal lattice of Bernal graphite, $\gamma-C$, is shown [3]. It corresponds to the standard diagram of its spatial symmetry group $P 6_{3} / m m c\left(D_{6 h}^{4}\right)$ [4]. In Fig $1, b$, the arrangement and orientation of the symmetry elements for the point group $6 / \mathrm{mmm}\left(D_{6 h}\right)$ are demonstrated.

Figure 2 illustrates the Brillouin zone in $\gamma-C$ crystals and its symmetry points. The points are denoted by letters corresponding to Herring's notation for hexagonal structures $[5,6]$.

The Wigner-Seitz unit cell and the Brillouin zone for single-layer graphene $C_{L, 1}$ are depicted in Fig. 3, $a$ and Fig. 3, b, respectively. Solid lines are used in Fig. 3, a to schematically mark the unit cell of graphene $C_{L, 1}$. The figure also illustrates the corresponding primitive translation vectors $\boldsymbol{a}_{1}$ and $\boldsymbol{a}_{2}$, as well as the orientation of cell symmetry elements in the three-dimensional space, which were used in calculations. The dashed lines in Fig. 3, a demonstrate the reciprocal lattice vectors $\boldsymbol{b}_{1}$ and $\boldsymbol{b}_{2}$ on an arbi-

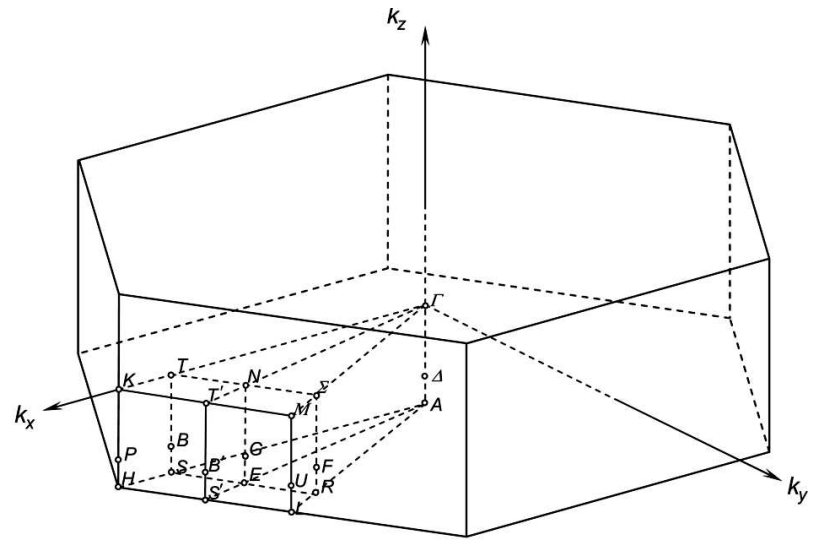

Fig. 2. Brillouin zone of graphite $\gamma-C$ crystals and its symmetry points (reproduced from work [1])

trary scale and the positions of reciprocal lattice sites in the reciprocal space. In Fig. 3, b, on the contrary, solid lines are used to show the reciprocal lattice vectors, and the dashed ones to demonstrate the direct lattice vectors. The unit cells (the Wigner-Seitz cells) of the graphene layer in the coordinate (Fig. 3,a) and reciprocal (Fig. 3, b) spaces (in the latter case, this cell coincides with the first Brillouin zone) are colored grey. In Fig. 3, b, the high-symmetry points $\Gamma$, $K$, and $M$ in the Brillouin zone of graphene are also 


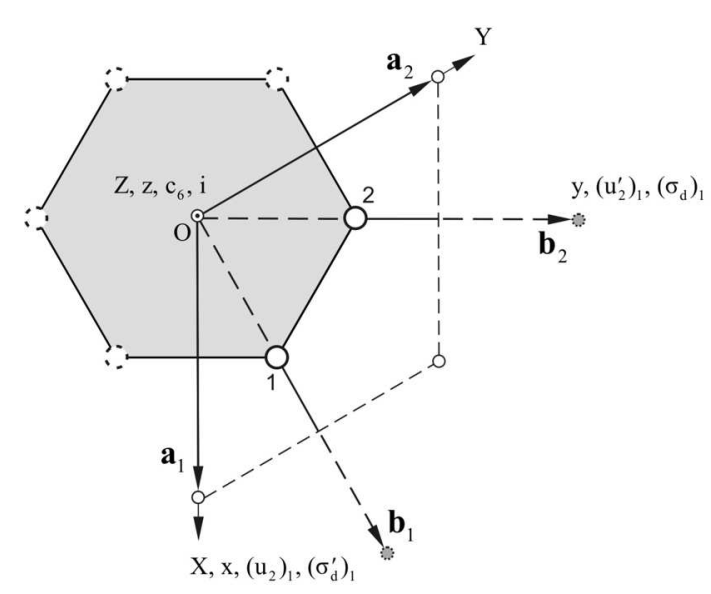

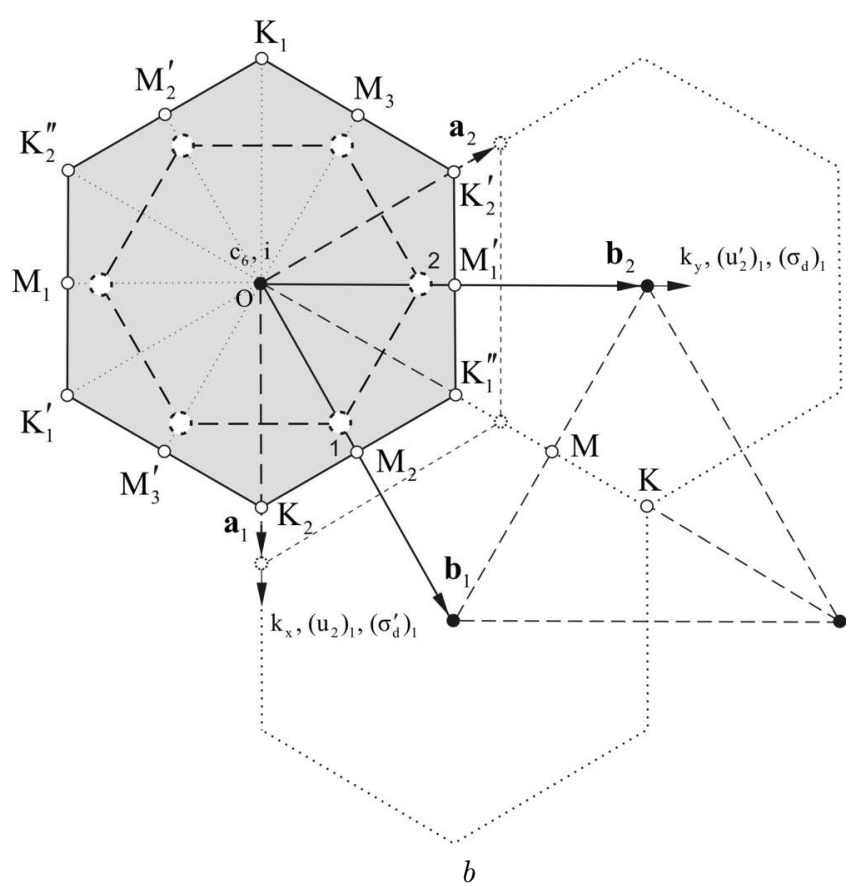

$b$

Fig. 3. Wigner-Seitz unit cell $(a)$ and Brillouin zone of single-layer graphene $C_{L, 1}(b)$ (reproduced from work [1])

shown. The equivalent points are marked by one or two primes.

The spatial symmetry group of the crystalline lattice of graphite, $P 6_{3} / m m c\left(D_{6 h}^{4}\right)$, is nonsymmorphic. It is determined by the basic (main) elements, which can be chosen as follows:

$h_{1}=(0 \mid e), h_{2}=\left(0 \mid c_{3}\right), h_{3}=\left(0 \mid c_{3}^{2}\right), h_{4}=\left(0 \mid\left(u_{2}\right)_{1}\right)$,

$h_{5}=\left(0 \mid\left(u_{2}\right)_{2}\right), h_{6}=\left(0 \mid\left(u_{2}\right)_{3}\right), h_{7}=\left(\frac{\boldsymbol{a}_{1}}{2} \mid c_{2}\right)$,

$h_{8}=\left(\frac{\boldsymbol{a}_{1}}{2} \mid c_{6}^{5}\right), h_{9}=\left(\frac{\boldsymbol{a}_{1}}{2} \mid c_{6}\right), h_{10}=\left(\frac{\boldsymbol{a}_{1}}{2} \mid\left(u_{2}^{\prime}\right)_{1}\right)$,

$h_{11}=\left(\frac{\boldsymbol{a}_{1}}{2} \mid\left(u_{2}^{\prime}\right)_{2}\right), h_{12}=\left(\frac{\boldsymbol{a}_{1}}{2} \mid\left(u_{2}^{\prime}\right)_{3}\right), h_{13}=(0 \mid i)$,

$h_{14}=\left(0 \mid i c_{3}\right), h_{15}=\left(0 \mid i c_{3}^{2}\right), h_{16}=\left(0 \mid i\left(u_{2}\right)_{1}\right)$,

$h_{17}=\left(0 \mid i\left(u_{2}\right)_{2}\right), h_{18}=\left(0 \mid i\left(u_{2}\right)_{3}\right), h_{19}=\left(\frac{\boldsymbol{a}_{1}}{2} \mid i c_{2}\right)$,

$h_{20}=\left(\frac{\boldsymbol{a}_{1}}{2} \mid i c_{6}^{5}\right), h_{21}=\left(\frac{\boldsymbol{a}_{1}}{2} \mid i c_{6}\right), h_{22}=\left(\frac{\boldsymbol{a}_{1}}{2} \mid i\left(u_{2}^{\prime}\right)_{1}\right)$,

$h_{23}=\left(\frac{\boldsymbol{a}_{1}}{2} \mid i\left(u_{2}^{\prime}\right)_{2}\right), h_{24}=\left(\frac{\boldsymbol{a}_{1}}{2} \mid\left(u_{2}^{\prime}\right)_{3}\right)$,

where $\boldsymbol{a}_{1}$ is a primitive vector of the crystal lattice directed along the axis $O Z(O z)$. At the same time,

344 the spatial symmetry group of the crystal lattice of single-layer graphene, $P 6 / \mathrm{mmm}(D G 80)$ [7], whose diagram coincides with that of the tri-periodic spatial group $P 6 / \mathrm{mmm}\left(D_{6 h}^{1}\right)$, is symmorphic, and all its "rotational" elements-the symmetry elements of the point group $6 / \mathrm{mmm}\left(D_{6 h}\right)$-do not contain nontrivial (partial) translations.

3. Qualitative Character of the Influence of Electron Spin and Time-Reversal Symmetry on the Energy Spectra of Elementary Excitations in Crystalline Graphite, Their Dispersion at the Points Along the Lines $\Gamma-\Delta-A$, $\boldsymbol{K}-\boldsymbol{P}-\boldsymbol{H}$, and $\boldsymbol{M}-\boldsymbol{U}-\boldsymbol{L}$ of Its Brillouin Zone, and the Energy Spectra and Dispersion of Electron $\pi$-Bands of Single-Layer Graphene at Points $\Gamma, K$, and $M$

3.1. Line $\Gamma-\Delta-A$ of crystalline graphite and point $\Gamma$ of single-layer graphene

\subsubsection{Points $\Gamma$}

At points $\Gamma$, the factor groups of the wave-vector groups with respect to the subgroups of trivial translations are isomorphic to the same point symmetry 
group $6 / m m m,\left(D_{6 h}\right)$ for both crystalline graphite $\gamma$ $C$ and single-layer graphene $C_{L, 1}$. This point group is a symmetry group of equivalent directions in the both structures: in crystalline graphite $\gamma-C$, where it is a group of the crystalline class, and in single-layer graphene $C_{L, 1}$, where it characterizes the symmetry of the macromolecular class [1].

The wave functions of vibrational elementary excitations at points $\Gamma-\Gamma_{\text {vib }}\left(\Gamma_{\text {lat.vib }}\right)$ are transformed, for both crystalline graphite and single-layer graphene, by the single-valued vector irreducible representations of the point group $6 / \mathrm{mmm}\left(D_{6 h}\right)$, which are irreducible representations of the projective class $K_{0}$ of this group. The representations of those excitations are determined by the formula [8]

$\Gamma_{\text {vib }}=\Gamma_{\text {eq }} \otimes \Gamma_{\text {vector }}$,

where $\Gamma_{\text {eq }}$ is the atomic equivalence representation ${ }^{1}$ at point $\Gamma$, and $\Gamma_{\text {vector }}$ is the representation of a polar vector $\mathbf{r}$ with the components $x, y$, and $z$.

Among the electron excitations at points $\Gamma$, we will consider only the excitations of electron $\pi$-bands, whose wave functions are orthogonal to those of the $s p^{2}$-hybridized $\sigma$-bands (i.e. the bands of $s p^{2}$ hybridized $\sigma$-electrons). In work [1], when determining the representations $\Gamma_{\pi}$ for states neglecting the spin (at a weak spin-orbit interaction), we used the following formula, which, in addition to the representation $\Gamma_{\text {eq }}$, includes only the representation $\Gamma_{z}$ :

$\Gamma_{\pi}=\Gamma_{\mathrm{eq}} \otimes \Gamma_{z}$,

where $\Gamma_{z}$ is a representation that is an irreducible representation of the group $6 / \mathrm{mmm}\left(D_{6 h}\right)$ for a vector directed along the $z$-axis, because the electron $\pi$-bands in graphite and graphene are formed by the nondegenerate electron orbitals $p_{z}$.

In order to determine the electron representation of $\pi$-bands making allowance for the electron spin, $\Gamma_{\pi}^{\prime}$, we have to use the formula

$\Gamma_{\pi}^{\prime}=\Gamma_{\mathrm{eq}} \otimes \Gamma_{z}^{\prime}$.

Here, $\Gamma_{z}^{\prime}$ is the representation of the electron $\pi$-orbital taking the spin into account. It is determined using

\footnotetext{
1 A technique used for determining the character of the atomic equivalence representations and the results of corresponding calculations for the high-symmetry points in the Brillouin zones of graphite and single-layer graphene were presented in work [1].
}

the formula

$\Gamma_{z}^{\prime}=\Gamma_{z} \otimes D_{1 / 2}^{+}$

In turn, $D_{1 / 2}^{+}$is an even two-dimensional (spinor) representation of the rotation group for the quantum number of total electron angular momentum $j=\frac{1}{2}$. Its characters in the case of the rotation by the angle $\phi$ are equal to [9]

$\chi_{j}\left(c_{\phi}\right)=\frac{\sin \left[\left(j+\frac{1}{2}\right) \phi\right]}{\sin \left(\frac{\phi}{2}\right)}$.

In Table 1 , the irreducible representations of the projective class $K_{0}$ for the group $6 / \mathrm{mmm}\left(D_{6 h}\right)$ are given. They describe the symmetry of vibrational and electron excitations at points $\Gamma$ of crystalline graphite $\gamma-C$ and single-layer graphene $C_{L, 1}$ making no allowance for the spin. They are identical to the ordinary single-valued vector irreducible representations. In addition, Table 1 contains the irreducible representations of the projective class $K_{1}$ for the group $6 / \mathrm{mmm}\left(D_{6 h}\right)$. They characterize the symmetry of electron states making allowance for the spin. They are two-valued spinors.

In Table 2, the characters of equivalence representations, $\Gamma_{\text {eq }}$, and the characters of representations that characterize the symmetry of electron $\pi$ bands making allowance for the electron spin $\left(\pi^{\prime}-\right.$ bands), $\Gamma_{\pi}^{\prime}=\Gamma_{\text {eq }} \otimes \Gamma_{z}^{\prime}$, are presented for points $\Gamma$ in the Brillouin zones of crystalline graphite $\gamma-C$ and single-layer graphene $C_{L, 1}$. The table also presents the characters of the representations $\Gamma_{z}, D_{1 / 2}^{+}$, and $\Gamma_{z}^{\prime}=\Gamma_{z} \otimes D_{1 / 2}^{+}$, as well as the corresponding projective representations, for other high-symmetry points in the Brillouin zones of those structures.

The electron excitation distributions at the highsymmetry points in the Brillouin zones of crystalline graphite and single-layer graphene with respect to irreducible two-valued spinor projective representations are shown in Table 3. For the sake of comparison, the distributions of electron excitations with respect to irreducible projective representations for $\pi$ bands without taking the electron spin into consideration are also included.

In Table 3, the following notations for the irreducible projective representations are applied in order to clearly distinguish two-valued spinor representations in various projective classes for various points 
Table 1. Characters of the one- and two-valued irreducible projective representations for points $\Gamma$ in the Brillouin zones of crystalline graphite $\gamma-C$ and single-layer graphene $C_{L, 1}$

\begin{tabular}{|c|c|c|c|c|c|c|c|c|c|c|c|c|c|c|c|c|c|c|}
\hline \multirow{2}{*}{$\begin{array}{c}\text { Projec- } \\
\text { tive } \\
\text { class }\end{array}$} & \multirow{2}{*}{\multicolumn{2}{|c|}{$\begin{array}{l}\text { Notation for } \\
\text { irreducible } \\
\text { projective } \\
\text { representation }\end{array}$}} & \multicolumn{16}{|c|}{$6 / m m m\left(D_{6 h}\right)$} \\
\hline & & & $e$ & $c_{3}$ & $c_{3}^{2}$ & $3 u_{2}$ & $c_{2}$ & $c_{6}^{5}$ & $c_{6}$ & $3 u_{2}^{\prime}$ & $i$ & $i c_{3}$ & $i c_{3}^{2}$ & $3 i u_{2}$ & $i c_{2}$ & $i c_{6}^{5}$ & $i c_{6}$ & $3 i u_{2}^{\prime}$ \\
\hline \multirow[t]{12}{*}{$K_{0}$} & $\Gamma_{1}^{+}$ & $A_{1}^{+}$ & 1 & 1 & 1 & 1 & 1 & 1 & 1 & 1 & 1 & 1 & 1 & 1 & 1 & 1 & 1 & 1 \\
\hline & $\Gamma_{1}^{-}$ & $A_{1}^{-}$ & 1 & 1 & 1 & 1 & 1 & 1 & 1 & 1 & -1 & -1 & -1 & -1 & -1 & -1 & -1 & -1 \\
\hline & & $A_{2}^{+}$ & 1 & 1 & 1 & 1 & -1 & -1 & -1 & -1 & 1 & 1 & 1 & 1 & -1 & -1 & -1 & -1 \\
\hline & $\Gamma_{2}^{-}$ & $A_{2}^{-}$ & 1 & 1 & 1 & 1 & -1 & -1 & -1 & -1 & -1 & -1 & -1 & -1 & 1 & 1 & 1 & 1 \\
\hline & $\Gamma_{3}^{+}$ & $A_{3}^{+}$ & 1 & 1 & 1 & -1 & 1 & 1 & 1 & -1 & 1 & 1 & 1 & -1 & 1 & 1 & 1 & -1 \\
\hline & $\Gamma_{3}^{-}$ & $A_{3}^{-}$ & 1 & 1 & 1 & -1 & 1 & 1 & 1 & -1 & -1 & -1 & -1 & 1 & -1 & -1 & -1 & 1 \\
\hline & $\Gamma_{4}^{+}$ & $A_{4}^{+}$ & 1 & 1 & 1 & -1 & -1 & -1 & -1 & 1 & 1 & 1 & 1 & -1 & -1 & -1 & -1 & 1 \\
\hline & $\Gamma_{4}^{-}$ & $A_{4}^{-}$ & 1 & 1 & 1 & -1 & -1 & -1 & -1 & 1 & -1 & -1 & -1 & 1 & 1 & 1 & 1 & -1 \\
\hline & $\Gamma_{5}^{+}$ & $E_{1}^{+}$ & 2 & -1 & -1 & 0 & 2 & -1 & -1 & 0 & 2 & -1 & -1 & 0 & 2 & -1 & -1 & 0 \\
\hline & $\Gamma_{5}^{-}$ & $E_{1}^{-}$ & 2 & -1 & -1 & 0 & 2 & -1 & -1 & 0 & -2 & 1 & 1 & 0 & -2 & 1 & 1 & 0 \\
\hline & $\Gamma_{6}^{+}$ & $E_{2}^{+}$ & 2 & -1 & -1 & 0 & -2 & 1 & 1 & 0 & 2 & -1 & -1 & 0 & -2 & 1 & 1 & 0 \\
\hline & & $E_{2}^{-}$ & 2 & -1 & -1 & 0 & -2 & 1 & 1 & 0 & -2 & 1 & 1 & 0 & 2 & -1 & -1 & 0 \\
\hline \multirow[t]{6}{*}{$K_{1}$} & $\Gamma_{7}^{+}$ & $\left(E_{1}^{\prime}\right)^{+}$ & 2 & 1 & -1 & 0 & 0 & $\sqrt{3}$ & $-\sqrt{3}$ & 0 & 2 & 1 & -1 & 0 & 0 & $\sqrt{3}$ & $-\sqrt{3}$ & 0 \\
\hline & & $\left(E_{1}^{\prime}\right)^{-}$ & 2 & 1 & -1 & 0 & 0 & $\sqrt{3}$ & $-\sqrt{3}$ & 0 & -2 & -1 & 1 & 0 & 0 & $-\sqrt{3}$ & $\sqrt{3}$ & 0 \\
\hline & & $\left(E_{2}^{\prime}\right)^{+}$ & 2 & 1 & -1 & 0 & 0 & $-\sqrt{3}$ & $\sqrt{3}$ & 0 & 2 & 1 & -1 & 0 & 0 & $-\sqrt{3}$ & $\sqrt{3}$ & 0 \\
\hline & & $\left(E_{2}^{\prime}\right)^{-}$ & 2 & 1 & -1 & 0 & 0 & $-\sqrt{3}$ & $\sqrt{3}$ & 0 & -2 & -1 & 1 & 0 & 0 & $\sqrt{3}$ & $-\sqrt{3}$ & 0 \\
\hline & $\Gamma_{9}^{+}$ & $\left(E_{3}^{\prime}\right)^{+}$ & 2 & -2 & 2 & 0 & 0 & 0 & 0 & 0 & 2 & -2 & 2 & 0 & 0 & 0 & 0 & 0 \\
\hline & & $\left(E_{3}^{\prime}\right)^{-}$ & 2 & -2 & 2 & 0 & 0 & 0 & 0 & 0 & -2 & 2 & -2 & 0 & 0 & 0 & 0 & 0 \\
\hline
\end{tabular}

in the Brillouin zones: letters denote points in the Brillouin zone of the structure; primed letters denote two-valued spinor representations, whereas nonprimed letters denote ordinary single-valued vector ones; parenthesized figures in the superscript indicate projective classes; figures in the internal subscript mean the ordinal number of an irreducible representation in the given projective class; and the signs "+" and "-" in the external superscript indicate the representation parity. It is evident that in those notations, $\left(\left(\Gamma^{\prime}\right)_{1}^{(1)}\right)^{+} \equiv \Gamma_{7}^{+}$and $\left(\left(\Gamma^{\prime}\right)_{2}^{(1)}\right)^{-} \equiv \Gamma_{8}^{-}$, where $\Gamma_{7}^{+}$and $\Gamma_{8}^{-}$are the spinor representations (written in the conventional notation system) that were used, e.g., to denote two-valued spinor representations in Table 1. An additional external subscript, if any, indicates the ordinal number of the representation, if there are several ones.

\subsubsection{Point $A$}

The wave-vector star of point $A$ in the Brillouin zone of crystalline graphite $\gamma-C$, similarly to that of point $\Gamma$, is composed of a single vector $\boldsymbol{k}_{A}=-\frac{1}{2} \boldsymbol{b}_{1}[1]$. The factor group of the wave-vector group with respect to the invariant translation subgroup for graphite crystals is, as it takes place for point $\Gamma$, also isomorphic to the group $6 / \mathrm{mmm}\left(D_{6 h}\right)$.

It was shown in work [1] that the two-valued (spinor) irreducible projective representations at point $A$ in the Brillouin zone of crystalline graphite $\gamma-C$ belong to the projective class $K_{4}$ of the point symmetry group of equivalent directions $F_{\boldsymbol{k}}, 6 / \mathrm{mmm}\left(D_{6 h}\right)$, coinciding with the crystal class group. It is so because the single-valued (vector) projective representations for point $A$, which are determined by the properties of the spatial symmetry group of graphite, $\mathrm{P}_{3} / \mathrm{mmc}\left(D_{6 h}\right)$, at this point, belong to the projective class $K_{5}$ [1], the transformation of spinors at symmetry operations of the directional groups of wave-vector groups to the projective class $K_{1}$, and the product of the projective classes $K_{5}$ and $K_{1}$, which is determined by the pairwise multiplication of the values of the coefficients $\alpha, \beta$, and $\gamma$ (i.e. $\alpha_{(5)} \alpha_{(1)}, \beta_{(5)} \beta_{(1)}$, and $\gamma_{(5)} \gamma_{(1)}$ ), equals $K_{5} K_{1}=$ $K_{4}$ in the system of notations used for projective classes [1].

ISSN 2071-0194. Ukr. J. Phys. 2020. Vol. 65, No. 4 
Table 2. Characters of the equivalence representations $D_{\text {eq }}$, the representations $D_{z}$ characterizing the spatial symmetry of $\pi$-orbitals, the spinor representations $D_{1 / 2}^{+}$, and the spinor representations $D_{z}^{\prime}=D_{z} \times D_{1 / 2}^{+}$characterizing the symmetry of $\pi$-orbitals taking the spin into account (the spin $\pi$-orbitals); and the characters of the representations $D_{\pi}^{\prime}$ that characterize the symmetry of electron $\pi$-bands taking the spin into account for various points of Brillouin zones in crystalline graphite $\gamma-C$ and single-layer graphene $C_{L, 1}$

Points $\Gamma$

Point groups $6 / \mathrm{mmm}\left(D_{6 h}\right)$

Projective classes $K_{1}$ except for the representations $\Gamma_{\mathrm{eq}}$ and $\Gamma_{z}$

\begin{tabular}{|c|c|c|c|c|c|c|c|c|c|c|c|c|c|c|c|c|c|c|}
\hline \multicolumn{3}{|c|}{$6 / m m m\left(D_{6 h}\right)$} & $e$ & $c_{3}$ & $c_{3}^{2}$ & $3 u_{2}$ & $c_{2}$ & $c_{6}^{5}$ & $c_{6}$ & $3 u_{2}^{\prime}$ & $i$ & $i c_{3}$ & $i c_{3}^{2}$ & $3 i u_{2}$ & $i c_{2}$ & $i c_{6}^{5}$ & $i c_{6}$ & $3 i u_{2}^{\prime}$ \\
\hline$\gamma-C$ & $\boldsymbol{k}=0$ & $\Gamma_{\text {eq }}$ & 4 & 4 & 4 & 0 & 0 & 0 & 0 & 4 & 0 & 0 & 0 & 4 & 4 & 4 & 4 & 0 \\
\hline & & $\Gamma_{z}$ & 1 & 1 & 1 & -1 & 1 & 1 & 1 & -1 & -1 & -1 & -1 & 1 & -1 & -1 & -1 & 1 \\
\hline & & $D_{1 / 2}^{+}$ & 2 & 1 & -1 & 0 & 0 & $-\sqrt{3}$ & $\sqrt{3}$ & 0 & 2 & 1 & -1 & 0 & 0 & $-\sqrt{3}$ & $\sqrt{3}$ & 0 \\
\hline & & $\Gamma_{z}^{\prime}$ & 2 & 1 & -1 & 0 & 0 & $-\sqrt{3}$ & $\sqrt{3}$ & 0 & -2 & -1 & 1 & 0 & 0 & $\sqrt{3}$ & $-\sqrt{3}$ & 0 \\
\hline & & $\Gamma_{\pi}^{\prime}$ & 8 & 4 & -4 & 0 & 0 & 0 & 0 & 0 & 0 & 0 & 0 & 0 & 0 & $4 \sqrt{3}$ & $-4 \sqrt{3}$ & 0 \\
\hline$C_{L, 1}$ & $\boldsymbol{k}=0$ & $\Gamma_{\text {eq }}$ & 2 & 2 & 2 & 0 & 0 & 0 & 0 & 2 & 0 & 0 & 0 & 2 & 2 & 2 & 2 & 0 \\
\hline & & $\Gamma_{\pi}^{\prime}$ & 4 & 2 & -2 & 0 & 0 & 0 & 0 & 0 & 0 & 0 & 0 & 0 & 0 & $2 \sqrt{3}$ & $-2 \sqrt{3}$ & 0 \\
\hline
\end{tabular}

Point $A$

Point group $6 / \mathrm{mmm}\left(D_{6 h}\right)$

Projective class $K_{4}$ except for the representation $A_{\text {eq }}$ and $A_{z}^{\prime}$

\begin{tabular}{|c|c|c|c|c|c|c|c|c|c|c|c|c|c|c|c|c|c|c|}
\hline \multicolumn{3}{|c|}{$6 / m m m\left(D_{6 h}\right)$} & $e$ & $c_{3}$ & $c_{3}^{2}$ & $3 u_{2}$ & $c_{2}$ & $c_{6}^{5}$ & $c_{6}$ & $3 u_{2}^{\prime}$ & $i$ & $i c_{3}$ & $i c_{3}^{2}$ & $3 i u_{2}$ & $i c_{2}$ & $i c_{6}^{5}$ & $i c_{6}$ & $3 i u_{2}^{\prime}$ \\
\hline$\gamma-C$ & $\begin{array}{c}\boldsymbol{k}_{A}= \\
=-(1 / 2) \boldsymbol{b}_{1}\end{array}$ & $\begin{array}{c}A_{\mathrm{eq}} \\
A_{z}^{\prime} \\
A_{\pi}^{\prime}\end{array}$ & $\begin{array}{l}4 \\
2 \\
8\end{array}$ & $\begin{array}{l}4 \\
1 \\
4\end{array}$ & $\begin{array}{c}4 \\
-1 \\
-4\end{array}$ & $\begin{array}{l}0 \\
0 \\
0\end{array}$ & $\begin{array}{l}0 \\
0 \\
0\end{array}$ & $\begin{array}{c}0 \\
-\sqrt{3} \\
0\end{array}$ & $\begin{array}{c}0 \\
\sqrt{3} \\
0\end{array}$ & $\begin{array}{l}0 \\
0 \\
0\end{array}$ & $\begin{array}{c}0 \\
-2 \\
0\end{array}$ & $\begin{array}{c}0 \\
-1 \\
0\end{array}$ & $\begin{array}{l}0 \\
1 \\
0\end{array}$ & $\begin{array}{l}4 \\
0 \\
0\end{array}$ & $\begin{array}{l}0 \\
0 \\
0\end{array}$ & $\begin{array}{c}0 \\
\sqrt{3} \\
0\end{array}$ & $\begin{array}{c}0 \\
-\sqrt{3} \\
0\end{array}$ & $\begin{array}{l}0 \\
0 \\
0\end{array}$ \\
\hline
\end{tabular}

Points $K$

Point groups $\overline{6} m 2\left(D_{3 h}\right)$

Projective classes $K_{1}$ except for the representations $K_{\mathrm{eq}}, K_{z}$ and $K_{\pi}$

\begin{tabular}{|c|c|c|c|c|c|c|c|c|c|c|}
\hline \multicolumn{3}{|c|}{$\overline{6} m 2\left(D_{3 h}\right)$} & $e$ & $c_{3}$ & $c_{3}^{2}$ & $3 u_{2}$ & $i c_{2}$ & $i c_{6}^{5}$ & $i c_{6}$ & $3 i u_{2}^{\prime}$ \\
\hline \multirow[t]{6}{*}{$\gamma-C$} & $\left(\boldsymbol{k}_{K}\right)_{1}=-(1 / 3)\left(2 \boldsymbol{b}_{2}-\boldsymbol{b}_{3}\right)$ & $K_{\text {eq }}$ & 4 & 1 & 1 & 0 & 4 & 1 & 1 & 0 \\
\hline & $\left(\boldsymbol{k}_{K}\right)_{2}=(1 / 3)\left(2 \boldsymbol{b}_{2}-\boldsymbol{b}_{3}\right)$ & $K_{z}$ & 1 & 1 & 1 & -1 & -1 & -1 & -1 & 1 \\
\hline & & $K_{\pi}$ & 4 & 1 & 1 & 0 & -4 & -1 & -1 & 0 \\
\hline & & $D_{1 / 2}^{+}$ & 2 & 1 & -1 & 0 & 0 & $-\sqrt{3}$ & $\sqrt{3}$ & 0 \\
\hline & & $K_{z}^{\prime}$ & 2 & 1 & -1 & 0 & 0 & $\sqrt{3}$ & $-\sqrt{3}$ & 0 \\
\hline & & $K_{\pi}^{\prime}$ & 8 & 1 & -1 & 0 & 0 & $\sqrt{3}$ & $-\sqrt{3}$ & 0 \\
\hline \multirow[t]{3}{*}{$C_{L, 1}$} & $\left(\boldsymbol{k}_{K}\right)_{1}=-(1 / 3)\left(2 \boldsymbol{b}_{1}-\boldsymbol{b}_{2}\right)$ & $K_{\text {eq }}$ & 2 & -1 & -1 & 0 & 2 & -1 & -1 & 0 \\
\hline & $\left(\boldsymbol{k}_{K}\right)_{2}=(1 / 3)\left(2 \boldsymbol{b}_{1}-\boldsymbol{b}_{2}\right)$ & $K_{\pi}$ & 2 & -1 & -1 & 0 & -2 & 1 & 1 & 0 \\
\hline & & $K_{\pi}^{\prime}$ & 4 & -1 & 1 & 0 & 0 & $-\sqrt{3}$ & $\sqrt{3}$ & 0 \\
\hline
\end{tabular}

Point $H$

Point group $\overline{6} m 2\left(D_{3 h}\right)$

Projective classes $K_{1}\left(H_{\mathrm{eq}}, H_{\pi}, D_{1 / 2}^{+}\right.$and $\left.H_{z}^{\prime}\right)$ and $K_{0}\left(H_{z}^{\prime}\right)$

\begin{tabular}{|c|c|c|c|c|c|c|c|c|c|c|}
\hline \multicolumn{3}{|c|}{$\overline{6} m 2\left(D_{3 h}\right)$} & $e$ & $c_{3}$ & $c_{3}^{2}$ & $3 u_{2}$ & $i c_{2}$ & $i c_{6}^{5}$ & $i c_{6}$ & $3 i u_{2}^{\prime}$ \\
\hline$\gamma-C$ & $\begin{array}{l}\left(\boldsymbol{k}_{H}\right)_{1}=-(1 / 2) \boldsymbol{b}_{1}-(1 / 3)\left(2 \boldsymbol{b}_{2}-\boldsymbol{b}_{3}\right) \\
\left(\boldsymbol{k}_{H}\right)_{2}=-(1 / 2) \boldsymbol{b}_{1}+(1 / 3)\left(2 \boldsymbol{b}_{2}-\boldsymbol{b}_{3}\right)\end{array}$ & $\begin{array}{c}H_{\mathrm{eq}} \\
H_{z} \\
H_{\pi} \\
D_{1 / 2}^{+} \\
H_{z}^{\prime} \\
H_{\pi}^{\prime}\end{array}$ & $\begin{array}{l}4 \\
1 \\
4 \\
2 \\
2 \\
8\end{array}$ & $\begin{array}{l}1 \\
1 \\
1 \\
1 \\
1 \\
1\end{array}$ & $\begin{array}{c}1 \\
1 \\
1 \\
-1 \\
-1 \\
-1\end{array}$ & $\begin{array}{c}0 \\
-1 \\
0 \\
0 \\
0 \\
0\end{array}$ & $\begin{array}{c}0 \\
-1 \\
0 \\
0 \\
0 \\
0\end{array}$ & $\begin{array}{c}\sqrt{3} \\
-1 \\
-\sqrt{3} i \\
-\sqrt{3} \\
\sqrt{3} \\
3 i\end{array}$ & $\begin{array}{c}-\sqrt{3} \\
-1 \\
\sqrt{3} i \\
\sqrt{3} \\
-\sqrt{3} \\
3 i\end{array}$ & $\begin{array}{l}0 \\
1 \\
0 \\
0\end{array}$ \\
\hline
\end{tabular}

ISSN 2071-0194. Ukr. J. Phys. 2020. Vol. 65, No. 4 
Point $P$

Point group $3 m\left(C_{3 v}\right)$

Projective class $K_{0}$

\begin{tabular}{|c|c|c|c|c|c|c|}
\hline \multicolumn{3}{|c|}{$3 m\left(C_{3 v}\right)$} & $e$ & $c_{3}$ & $c_{3}^{2}$ & $3 i u_{2}^{\prime}$ \\
\hline$\gamma-C$ & $\begin{array}{l}\left(\boldsymbol{k}_{P}\right)_{1}=-\boldsymbol{k}_{z}-(1 / 3)\left(2 \boldsymbol{b}_{2}-\boldsymbol{b}_{3}\right) \\
\left(\boldsymbol{k}_{P}\right)_{2}=-\boldsymbol{k}_{z}+(1 / 3)\left(2 \boldsymbol{b}_{2}-\boldsymbol{b}_{3}\right) \\
\left(\boldsymbol{k}_{P}\right)_{3}=\boldsymbol{k}_{z}-(1 / 3)\left(2 \boldsymbol{b}_{2}-\boldsymbol{b}_{3}\right) \\
\left(\boldsymbol{k}_{P}\right)_{4}=\boldsymbol{k}_{z}+(1 / 3)\left(2 \boldsymbol{b}_{2}-\boldsymbol{b}_{3}\right)\end{array}$ & $\begin{array}{c}P_{\mathrm{eq}} \\
P_{z} \\
P_{\pi} \\
D_{1 / 2}^{+} \\
P_{z}^{\prime} \\
P_{\pi}^{\prime}\end{array}$ & $\begin{array}{l}4 \\
1 \\
4 \\
2 \\
2 \\
8\end{array}$ & $\begin{array}{l}1 \\
1 \\
1 \\
1 \\
1 \\
1\end{array}$ & $\begin{array}{c}1 \\
1 \\
1 \\
-1 \\
-1 \\
-1\end{array}$ & $\begin{array}{l}0 \\
1 \\
0 \\
0 \\
0 \\
0\end{array}$ \\
\hline
\end{tabular}

Points $M$

Point groups $m m m\left(D_{2 h}\right)$

Projective classes $K_{0}\left(M_{\mathrm{eq}}, M_{z}\right.$ and $\left.M_{\pi}\right)$ and $K_{1}\left(D_{1 / 2}^{+}, M_{z}^{\prime}\right.$ and $\left.M_{\pi}^{\prime}\right)$

\begin{tabular}{|c|c|c|c|c|c|c|c|c|c|c|}
\hline \multicolumn{3}{|c|}{$m m m\left(D_{2 h}\right)$} & $e$ & $\left(u_{2}\right)_{1}$ & $c_{2}$ & $\left(u_{2}^{\prime}\right)_{1}$ & $i$ & $i\left(u_{2}\right)_{1}$ & $i c_{2}$ & $i\left(u_{2}^{\prime}\right)_{1}$ \\
\hline \multirow{6}{*}{$\gamma-C$} & $\left(\boldsymbol{k}_{M}\right)_{1}=-(1 / 2) \boldsymbol{b}_{3}$ & $M_{\mathrm{eq}}$ & 4 & 0 & 0 & 4 & 0 & 4 & 4 & 0 \\
\hline & $\left(\boldsymbol{k}_{M}\right)_{2}=(1 / 2) \boldsymbol{b}_{2}$ & $M_{z}$ & 1 & -1 & 1 & -1 & -1 & 1 & -1 & 1 \\
\hline & $\left(\boldsymbol{k}_{M}\right)_{3}=-(1 / 2)\left(\boldsymbol{b}_{2}-\boldsymbol{b}_{3}\right)$, & $M_{\pi}$ & 4 & 0 & 0 & -4 & 0 & 4 & -4 & 0 \\
\hline & & $D_{1 / 2}^{+}$ & 2 & 0 & 0 & 0 & 2 & 0 & 0 & 0 \\
\hline & & $M_{z}^{\prime}$ & 2 & 0 & 0 & 0 & -2 & 0 & 0 & 0 \\
\hline & & $M_{\pi}^{\prime}$ & 8 & 0 & 0 & 0 & 0 & 0 & 0 & 0 \\
\hline \multirow[t]{3}{*}{$C_{L, 1}$} & $\left(\boldsymbol{k}_{M}\right)_{1}=-(1 / 2) \boldsymbol{b}_{2}$ & $M_{\mathrm{eq}}$ & 2 & 0 & 0 & 2 & 0 & 2 & 2 & 0 \\
\hline & $\left(\boldsymbol{k}_{M}\right)_{2}=(1 / 2) \boldsymbol{b}_{1}$ & $M_{\pi}$ & 2 & 0 & 0 & -2 & 0 & 2 & -2 & 0 \\
\hline & $\left(\boldsymbol{k}_{M}\right)_{3}=-(1 / 2)\left(\boldsymbol{b}_{1}-\boldsymbol{b}_{2}\right)$ & $M_{\pi}^{\prime}$ & 4 & 0 & 0 & 0 & 0 & 0 & 0 & 0 \\
\hline
\end{tabular}

Point $L$

Point group $m m m\left(D_{2 h}\right)$

Projective classes $K_{5}\left(L_{\pi}\right)$ and $K_{4}\left(L_{\pi}^{\prime}\right)$

\begin{tabular}{|c|c|c|c|c|c|c|c|c|c|c|}
\hline \multicolumn{3}{|c|}{$m m m\left(D_{2 h}\right)$} & $e$ & $\left(u_{2}\right)_{1}$ & $c_{2}$ & $\left(u_{2}^{\prime}\right)_{1}$ & $i$ & $i\left(u_{2}\right)_{1}$ & $i c_{2}$ & $i\left(u_{2}^{\prime}\right)_{1}$ \\
\hline \multirow[t]{6}{*}{$\gamma-C$} & $\left(\boldsymbol{k}_{L}\right)_{1}=-(1 / 2)\left(\boldsymbol{b}_{1}+\boldsymbol{b}_{3}\right)$ & $L_{\text {eq }}$ & 4 & 0 & 0 & 0 & 0 & 4 & 0 & 0 \\
\hline & $\left(\boldsymbol{k}_{L}\right)_{2}=-(1 / 2)\left(\boldsymbol{b}_{1}-\boldsymbol{b}_{2}\right)$ & $L_{z}$ & 1 & -1 & 1 & -1 & -1 & 1 & -1 & 1 \\
\hline & $\left(\boldsymbol{k}_{L}\right)_{3}=-(1 / 2)\left(\boldsymbol{b}_{1}+\boldsymbol{b}_{2}-\boldsymbol{b}_{3}\right)$ & $L_{\pi}$ & 4 & 0 & 0 & 0 & 0 & 4 & 0 & 0 \\
\hline & & $D_{1 / 2}^{+}$ & 2 & 0 & 0 & 0 & 2 & 0 & 0 & 0 \\
\hline & & $L_{z}^{\prime}$ & 2 & 0 & 0 & 0 & -2 & 0 & 0 & 0 \\
\hline & & $L_{\pi}^{\prime}$ & 8 & 0 & 0 & 0 & 0 & 0 & 0 & 0 \\
\hline
\end{tabular}

The factor-system for point $A$ with making allowance for the spin, $\omega_{2, A}\left(r_{2}, r_{1}\right)$, is the product of the factor-systems $\omega_{1, A}\left(r_{2}, r_{1}\right)$ and $\omega_{2}\left(r_{2}, r_{1}\right)$, i.e. $\omega_{2, A}\left(r_{2}, r_{1}\right)=\omega_{1, A}\left(r_{2}, r_{1}\right) \omega_{2}\left(r_{2}, r_{1}\right)$. The former is determined by the structure of the spatial group of a graphite crystal, $P 6_{3} / m m c\left(D_{6 h}^{4}\right)$, at point $A$ neglecting the spin, and the latter describes the transformations of spinors at point $\Gamma$ (in the point symmetry group $\left.6 / \mathrm{mmm}\left(D_{6 h}\right)\right)$. They and their structure are described in details in work [1] (see Tables 1 and 6 in the cited work). The factor-system $\omega_{2, A}\left(r_{2}, r_{1}\right)$ is presented in Table 4.
The standard factor-system for point $A$ with making allowance for the spin, $\omega_{2, A}^{\prime}\left(r_{2}, r_{1}\right)$, which belongs to the projective class $K_{4}$, coincides with the standard factor-system of this class, $\omega_{(4)}^{\prime}\left(r_{2}, r_{1}\right)$, and is the product of the standard factor-systems $\omega_{1, A}^{\prime}\left(r_{2}, r_{1}\right) \equiv$ $\equiv \omega_{(5)}^{\prime}\left(r_{2}, r_{1}\right)$ and $\omega_{2}^{\prime}\left(r_{2}, r_{1}\right)$, i.e. $\omega_{2, A}^{\prime}\left(r_{2}, r_{1}\right) \equiv$ $\equiv \omega_{(4)}^{\prime}\left(r_{2}, r_{1}\right)=\omega_{(5)}^{\prime}\left(r_{2}, r_{1}\right) \omega_{2}^{\prime}\left(r_{2}, r_{1}\right)$. This factorsystem is presented in Table 5 . The reduction coefficients $u_{2, A}(r)$ of the factor-system $\omega_{2, A}\left(r_{2}, r_{1}\right)$ to the standard form $\omega_{2, A}^{\prime}\left(r_{2}, r_{1}\right) \equiv \omega_{(4)}^{\prime}\left(r_{2}, r_{1}\right)$ are determined as the products of the corresponding reduction coefficients $u_{1, A}(r)$ and $u_{2}(r)$ of the factor- 
Table 3. Distributions of electron excitations over the irreducible projective representations of corresponding projective classes for electron $\pi$-bands at high-symmetry points in the Brillouin zones of the crystalline graphite $\gamma-C$ and single-layer graphene $C_{L, 1}$ structures not taking $(a)$ and taking $(b)$ the electron spin into account

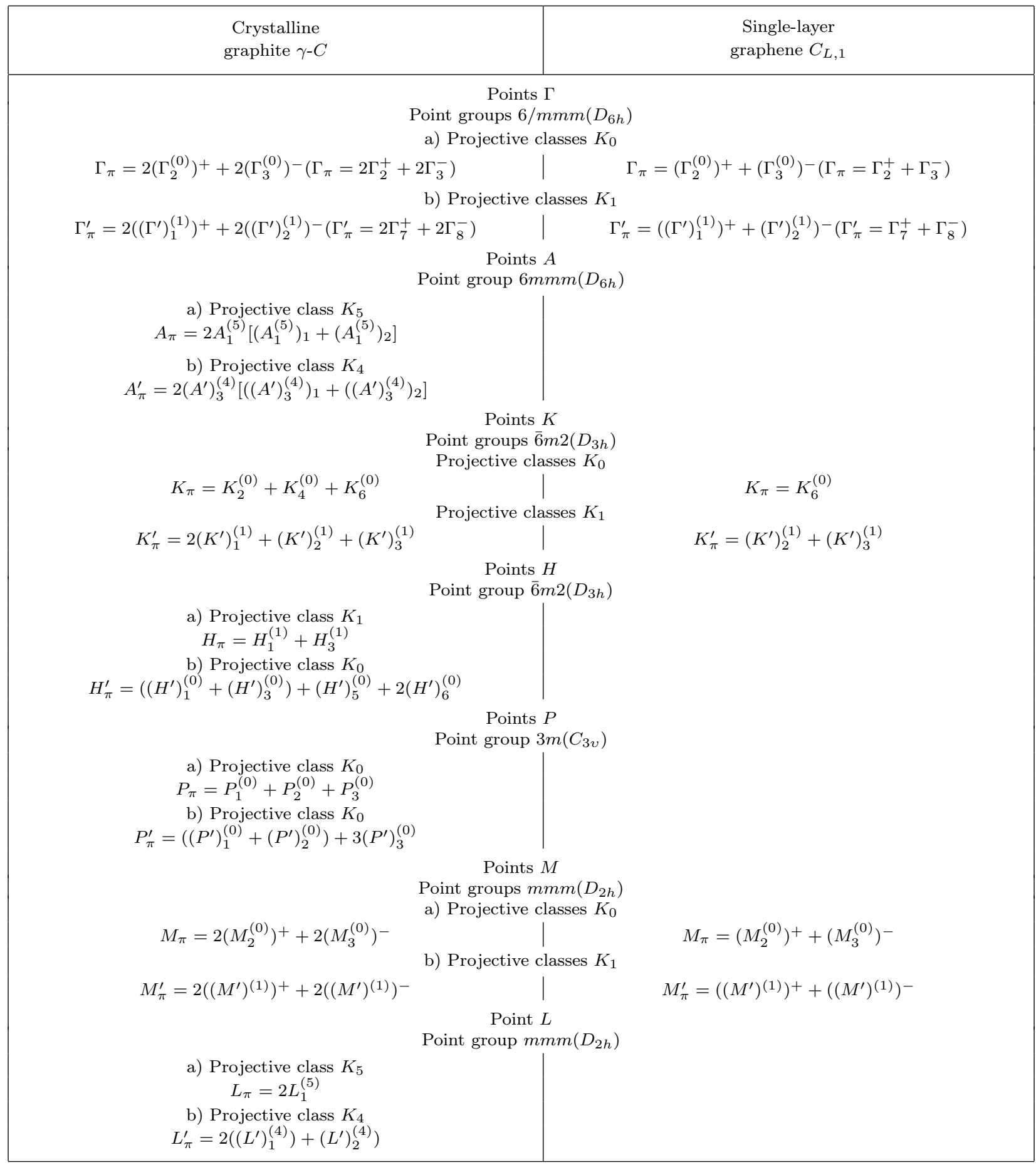




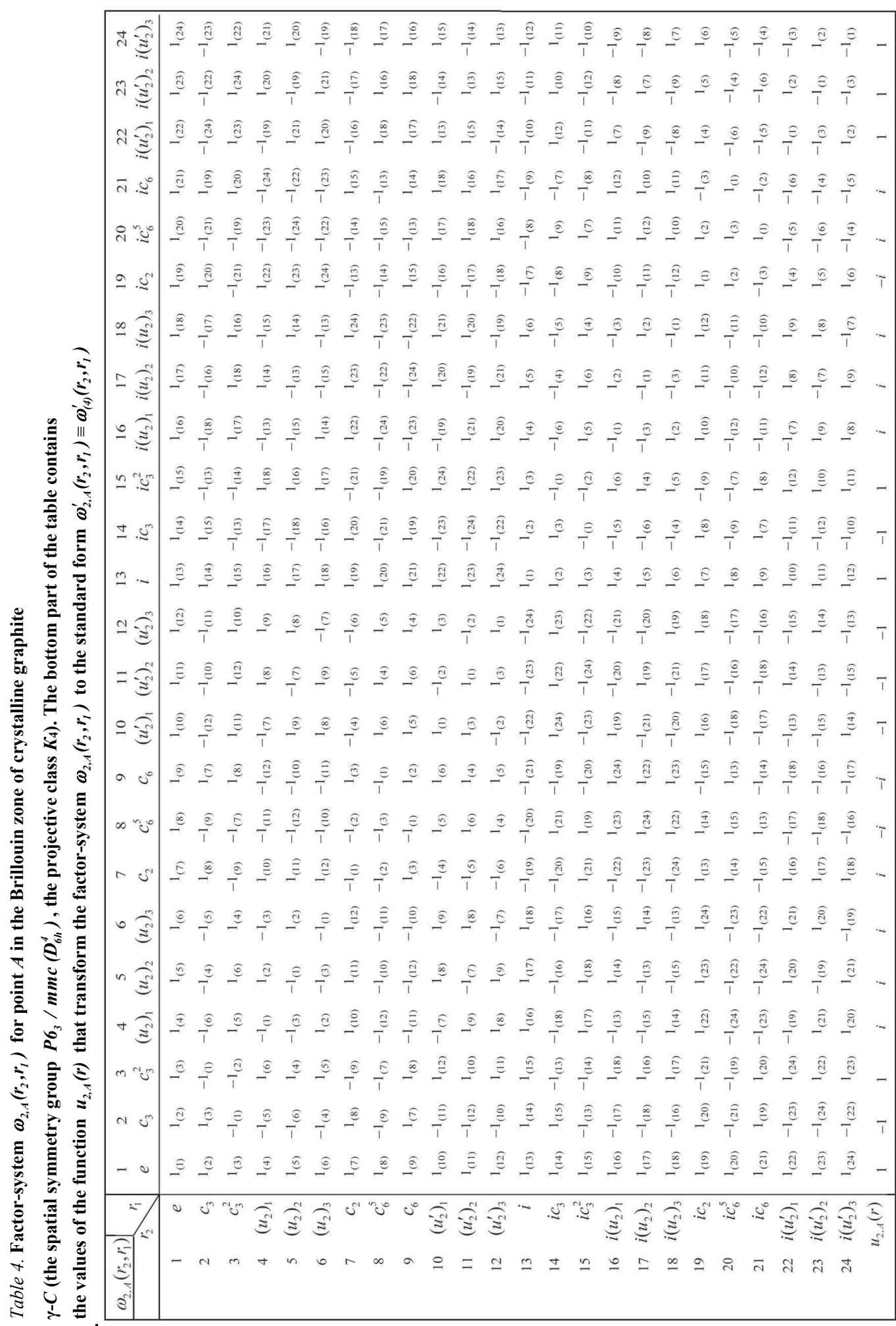




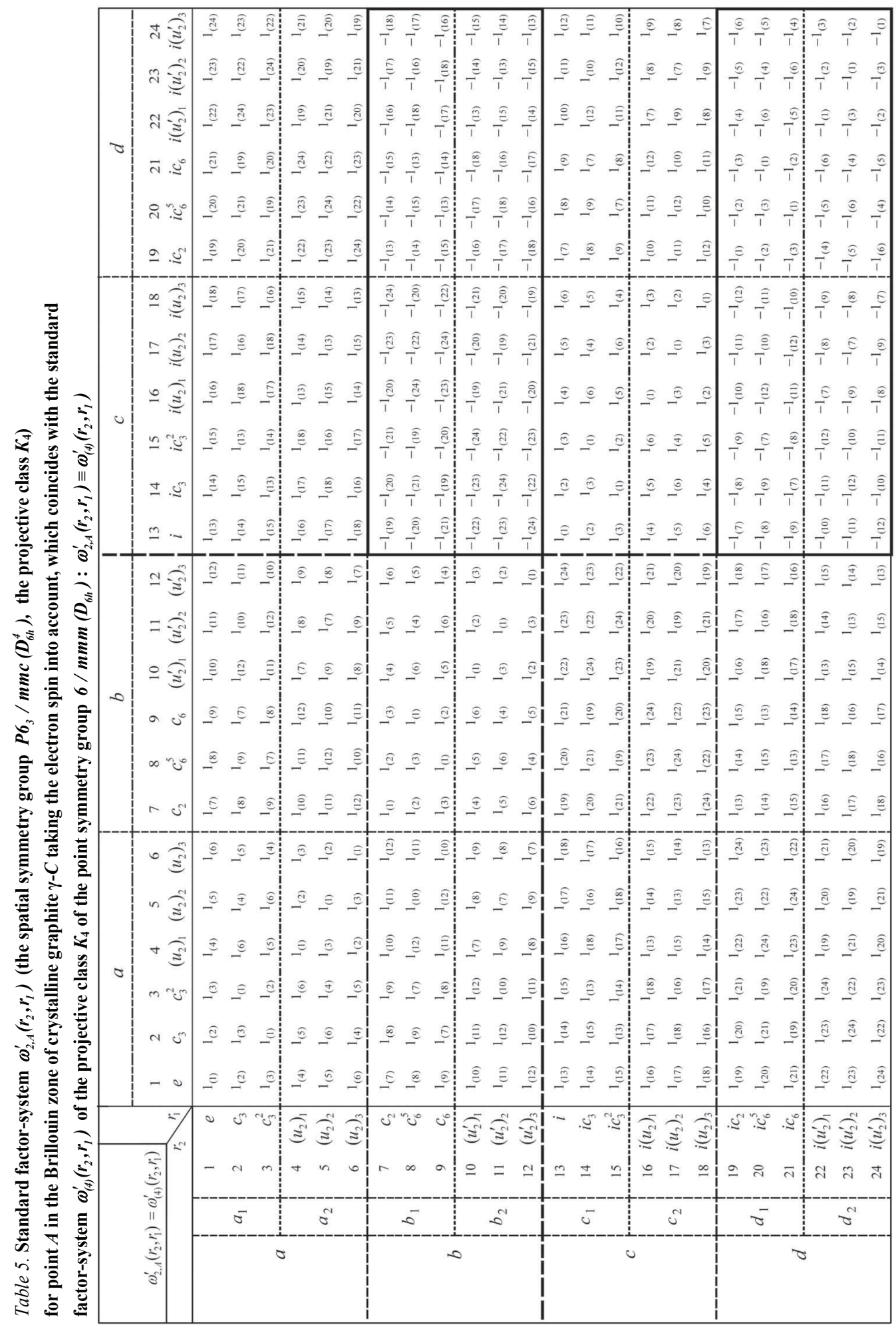


Table 6. Characters of the irreducible projective representations of the projective class $K_{4}$ corresponding to the standard factor-system $\omega_{(4)}^{\prime}\left(r_{2}, r_{1}\right)$ of this class

\begin{tabular}{|c|c|c|c|c|c|c|c|c|c|c|c|c|c|c|c|c|c|}
\hline \multirow{2}{*}{$\begin{array}{c}\text { Projec- } \\
\text { tive } \\
\text { class }\end{array}$} & \multirow{2}{*}{$\begin{array}{l}\text { Notation } \\
\text { for irreducible } \\
\text { projective } \\
\text { representation }\end{array}$} & \multicolumn{16}{|c|}{$6 / m m m\left(D_{6 h}\right)$} \\
\hline & & $e$ & $c_{1}$ & $c_{3}^{2}$ & $3 u_{2}$ & $c_{2}$ & $c_{6}^{5}$ & $c_{6}$ & $3 u_{2}^{\prime}$ & $i$ & $i c_{3}$ & $i c_{3}^{2}$ & $3 i u_{2}$ & $i c_{2}$ & $i c_{6}^{5}$ & $i c_{6}$ & $3 i u_{2}^{\prime}$ \\
\hline \multirow[t]{4}{*}{$K_{4}$} & $P_{1}^{(4)}$ & 2 & 2 & 2 & 2 & 0 & 0 & 0 & 0 & 0 & 0 & 0 & 0 & 0 & 0 & 0 & 0 \\
\hline & $P_{2}^{(4)}$ & 2 & 2 & 2 & -2 & 0 & 0 & 0 & 0 & 0 & 0 & 0 & 0 & 0 & 0 & 0 & 0 \\
\hline & $Q^{(4)}$ & 4 & -2 & -2 & 0 & 0 & 0 & 0 & 0 & 0 & 0 & 0 & 0 & 0 & 0 & 0 & 0 \\
\hline & $u_{2, A}(r)$ & 1 & -1 & 1 & $i$ & $i$ & $-i$ & $-i$ & -1 & 1 & -1 & 1 & $i$ & $-i$ & $i$ & $i$ & 1 \\
\hline
\end{tabular}

Table 7. Characters of the two-valued (spinor) irreducible projective representations of point $A$ in the Brillouin zone of crystalline graphite $\gamma-C$

\begin{tabular}{|c|c|c|c|c|c|c|c|c|c|c|c|c|c|c|c|c|c|}
\hline \multirow{2}{*}{$\begin{array}{l}\text { Projec- } \\
\text { tive } \\
\text { class }\end{array}$} & \multirow{2}{*}{$\begin{array}{l}\text { Notation } \\
\text { for irreducible } \\
\text { projective } \\
\text { representation }\end{array}$} & \multicolumn{16}{|c|}{$6 / m m m\left(D_{6 h}\right)$} \\
\hline & & $e$ & $c_{3}$ & $c_{3}^{2}$ & $3 u_{2}$ & $c_{2}$ & $c_{6}^{5}$ & $c_{6}$ & $3 u_{2}^{\prime}$ & $i$ & $i c_{3}$ & $i c_{3}^{2}$ & $3 i u_{2}$ & $i c_{2}$ & $i c_{6}^{5}$ & $i c_{6}$ & $3 i u_{2}^{\prime}$ \\
\hline \multirow[t]{3}{*}{$K_{4}$} & \multirow{3}{*}{$\begin{aligned}\left(\left(A^{\prime}\right)_{1}^{(4)}+\left(A^{\prime}\right)_{2}^{(4)}\right)< & \left(A^{\prime}\right)_{1}^{(4)} \\
& \left(A^{\prime}\right)_{2}^{(4)} \\
& \left(A^{\prime}\right)_{3}^{(4)}\end{aligned}$} & 2 & -2 & 2 & $2 i$ & 0 & 0 & 0 & 0 & 0 & 0 & 0 & 0 & 0 & 0 & 0 & 0 \\
\hline & & 2 & -2 & 2 & $-2 i$ & 0 & 0 & 0 & 0 & 0 & 0 & 0 & 0 & 0 & 0 & 0 & 0 \\
\hline & & 4 & 2 & -2 & 0 & 0 & 0 & 0 & 0 & 0 & 0 & 0 & 0 & 0 & 0 & 0 & 0 \\
\hline
\end{tabular}

systems $\omega_{1, A}\left(r_{2}, r_{1}\right)$ and $\omega_{2}\left(r_{2}, r_{1}\right)$ to the standard form, i.e. $u_{2, A}(r)=u_{1, A}(r) u_{2}(r)$ [1]. The values of the coefficients $u_{2, A}(r)$ are shown in the bottom part of Table 4.

The characters of the irreducible projective representations of the projective class $K_{4}$, which correspond to the standard factor-system of this class, $\omega_{(4)}^{\prime}\left(r_{2}, r_{1}\right)$, are given in Table 6 , and the characters of the two-valued (spinor) irreducible projective representations of point $A$ are quoted in Table 7 . In the bottom part of Table 6 , the values of the coefficients $u_{2, A}(r)$ are shown. As it has to be, the equalities

$\left(A^{\prime}\right)_{i}^{(4)}=u_{2, A}(r) P_{i}^{(4)}$

are satisfied.

The characters of the projective equivalence representation at point $A$, i.e. the representation $A_{\text {eq }}$ and the two-valued representations $A_{z}^{\prime} \equiv \Gamma_{z}^{\prime}$ and $A_{\pi}^{\prime}$ are given in Table 2 . The distributions of electron excitations at point $A$ in the Brillouin zone of crystalline graphite $\gamma$-C for the $\pi$-bands neglecting the electron spin and taking it into account are presented in Table 3. It is of interest that electron excitations of $\pi$ bands at point $A$ in the Brillouin zone of crystalline graphite $\gamma-C$ making allowance for the spin are fourfold degenerate, because their states are transformed according to the four-dimensional irreducible projective representations of the projective class $K_{4}$. In the absence of external magnetic fields, additional conditions associated with the time-reversal invariance are imposed on the wave functions of states and, accordingly, on the representations. In this case, some of the states may become additionally degenerate.

Let us account for the time-reversal invariance of the states at points $\Gamma$ and $A$ in the Brillouin zone of crystalline graphite and point $\Gamma$ in the Brillouin zone of single-layer graphene with the help of the Herring criterion $[5,6,9]$. The corresponding calculation procedure is described in works $[9,10]$ in detail. In particular, the summation is carried out over the elements $g^{\prime}=\left(\boldsymbol{\alpha} \mid r^{\prime}\right)$ of the wave-vector group $G_{\boldsymbol{k}}$ that satisfy the condition $g^{\prime} \boldsymbol{k}=-\boldsymbol{k}\left(r^{\prime} \boldsymbol{k}=-\boldsymbol{k}\right)$.

For points $\Gamma$ and $A$ of crystalline graphite $\gamma-C$ and point $\Gamma$ of single-layer graphene $C_{L, 1}$, each wavevector star has one ray. At those points, the wave vectors $-\boldsymbol{k}$ and $\boldsymbol{k}$ are equivalent $(-\boldsymbol{k} \equiv \boldsymbol{k})$, and the condition $g^{\prime} \boldsymbol{k}=-\boldsymbol{k}$ is satisfied, for crystalline graphite $\gamma-C$, by the elements $g_{1}^{\prime}=(0 \mid e), g_{2}^{\prime}=\left(0 \mid c_{3}\right)$,

ISSN 2071-0194. Ukr. J. Phys. 2020. Vol. 65, No. 4 
$g_{3}^{\prime}=\left(0 \mid c_{3}^{2}\right), g_{4}^{\prime}=\left(0 \mid\left(u_{2}\right)_{1}\right), g_{5}^{\prime}=\left(0 \mid\left(u_{2}\right)_{2}\right), g_{6}^{\prime}=$ $=\left(0 \mid\left(u_{2}\right)_{3}\right), g_{7}^{\prime}=\left(\frac{\boldsymbol{a}_{1}}{2} \mid c_{2}\right), g_{8}^{\prime}=\left(\frac{\boldsymbol{a}_{1}}{2} \mid c_{6}^{5}\right), g_{9}^{\prime}=$ $=\left(\frac{\boldsymbol{a}_{1}}{2} \mid c_{6}\right), g_{10}^{\prime}=\left(\frac{\boldsymbol{a}_{1}}{2} \mid\left(u_{2}^{\prime}\right)_{1}\right), g_{11}^{\prime}=\left(\frac{\boldsymbol{a}_{1}}{2} \mid\left(u_{2}^{\prime}\right)_{2}\right)$, $g_{12}^{\prime}=\left(\frac{\boldsymbol{a}_{1}}{2} \mid\left(u_{2}^{\prime}\right)_{3}\right), g_{13}^{\prime}=(0 \mid i), g_{14}^{\prime}=\left(0 \mid i c_{3}\right), g_{15}^{\prime}=$ $=\left(0 \mid i c_{3}^{2}\right), g_{16}^{\prime}=\left(0 \mid i\left(u_{2}\right)_{1}\right), g_{17}^{\prime}=\left(0 \mid i\left(u_{2}\right)_{2}\right), g_{18}^{\prime}=$ $=\left(0 \mid i\left(u_{2}\right)_{3}\right), g_{19}^{\prime}=\left(\frac{\boldsymbol{a}_{1}}{2} \mid i c_{2}\right), g_{20}^{\prime}=\left(\frac{\boldsymbol{a}_{1}}{2} \mid i c_{6}^{5}\right), g_{21}^{\prime}=$ $=\left(\frac{\boldsymbol{a}_{1}}{2} \mid i c_{6}\right), g_{22}^{\prime}=\left(\frac{\boldsymbol{a}_{1}}{2} \mid i\left(u_{2}^{\prime}\right)_{1}\right), g_{23}^{\prime}=\left(\frac{\boldsymbol{a}_{1}}{2} \mid i\left(u_{2}^{\prime}\right)_{2}\right)$, and $g_{24}^{\prime}=\left(\frac{a_{1}}{2} \mid i\left(u_{2}^{\prime}\right)_{3}\right)$ and, for single-layer graphene $C_{L, 1}$, by the same values, for which the nontrivial translation $\frac{a_{1}}{2}=0$, because the spatial group of single-layer graphene is symmorphic and, unlike the spatial group of crystalline graphite, does not contain nontrivial translations.

It is easy to calculate the squares of those elements, $\left(g^{\prime}\right)^{2}=\left(r \boldsymbol{\alpha}+\boldsymbol{\alpha} \mid r^{2}\right)$. In particular, for points $\Gamma$ and $A$ of crystalline graphite, $\left(g_{1}^{\prime}\right)^{2}=(0 \mid e)$, $\left(g_{2}^{\prime}\right)^{2}=\left(0 \mid c_{3}^{2}\right), \quad\left(g_{3}^{\prime}\right)^{2}=\left(0 \mid q c_{3}\right),\left(g_{4}^{\prime}\right)^{2}=(0 \mid q)$, $\left(g_{5}^{\prime}\right)^{2}=(0 \mid q),\left(g_{6}^{\prime}\right)^{2}=(0 \mid q),\left(g_{7}^{\prime}\right)^{2}=\left(\boldsymbol{a}_{1} \mid q\right)$, $\left(g_{8}^{\prime}\right)^{2}=\left(\boldsymbol{a}_{1} \mid q c_{3}^{2}\right),\left(g_{9}^{\prime}\right)^{2}=\left(\boldsymbol{a}_{1} \mid c_{3}\right),\left(g_{10}^{\prime}\right)^{2}=(0 \mid q)$, $\left(g_{11}^{\prime}\right)^{2}=(0 \mid q),\left(g_{12}^{\prime}\right)^{2}=(0 \mid q),\left(g_{13}^{\prime}\right)^{2}=(0 \mid e)$, $\left(g_{14}^{\prime}\right)^{2}=\left(0 \mid c_{3}^{2}\right),\left(g_{15}^{\prime}\right)^{2}=\left(0 \mid q c_{3}\right),\left(g_{16}^{\prime}\right)^{2}=(0 \mid q)$, $\left(g_{17}^{\prime}\right)^{2}=(0 \mid q),\left(g_{18}^{\prime}\right)^{2}=(0 \mid q),\left(g_{19}^{\prime}\right)^{2}=(0 \mid q)$, $\left(g_{20}^{\prime}\right)^{2}=\left(0 \mid q c_{3}^{2}\right),\left(g_{21}^{\prime}\right)^{2}=\left(0 \mid c_{3}\right),\left(g_{22}^{\prime}\right)^{2}=\left(\boldsymbol{a}_{1} \mid q\right)$, $\left(g_{23}^{\prime}\right)^{2}=\left(\boldsymbol{a}_{1} \mid q\right),\left(g_{24}^{\prime}\right)^{2}=\left(\boldsymbol{a}_{1} \mid q\right)$ where $q$ is the rotation by the angle $2 \pi$ around the corresponding axis; and, for point $\Gamma$ of single-layer graphene $C_{L, 1}$, these are the same values, for which the trivial translation vector $\boldsymbol{a}_{1}=0$.

The stages of calculations according to the Herring criterion and the results obtained are presented in Table 18 (see Appendix). From the values of the Herring criterion, it is easy to see that both the single- and two-valued projective representations at points $\Gamma$ and $A$ of crystalline graphite $\gamma-C$ and point $\Gamma$ of single-layer graphene $C_{L, 1}$, except for the representations $\left(A^{\prime}\right)_{1}^{(4)}$ and $\left(A^{\prime}\right)_{2}^{(4)}$ for crystalline graphite, are related to the case $a_{1}[9]$, where there is no additional degeneration of the states provided that their invariance with respect to the time reversal is taken into account. At the same time, the projective representations at point $A$ for crystalline graphite - these are the representations $\left(A^{\prime}\right)_{1}^{(4)}$ and $\left(A^{\prime}\right)_{2}^{(4)}$ - are related to the case $b_{1}[9]$ and, being representations with complex-conjugated characters, group together to increase the degeneracy order of electron states to four. This grouping of complex-conjugated representations is illustrated in Table 7.

\subsubsection{Point $\Delta$}

The group of equivalent directions of the wave-vector group at point $\Delta$ of crystalline graphite $\gamma-C$ is the group $6 m m\left(C_{6 v}\right)$. The wave-vector star at this point contains two rays. The irreducible single- and twovalued projective representations for point $\Delta$ are given in work [1]. The condition $g^{\prime} \boldsymbol{k}=-\boldsymbol{k}$ at this point is satisfied by the elements $g^{\prime} \boldsymbol{k}=-\boldsymbol{k}: g_{4}^{\prime}=$ $=\left(0 \mid\left(u_{2}\right)_{1}\right), g_{5}^{\prime}=\left(0 \mid\left(u_{2}\right)_{2}\right), g_{6}^{\prime}=\left(0 \mid\left(u_{2}\right)_{3}\right), g_{10}^{\prime}=$ $=\left(\frac{\boldsymbol{a}_{1}}{2} \mid\left(u_{2}^{\prime}\right)_{1}\right), g_{11}^{\prime}=\left(\frac{\boldsymbol{a}_{1}}{2} \mid\left(u_{2}^{\prime}\right)_{2}\right), g_{12}^{\prime}=\left(\frac{\boldsymbol{a}_{1}}{2} \mid\left(u_{2}^{\prime}\right)_{3}\right)$, $g_{13}^{\prime}=(0 \mid i), g_{14}^{\prime}=\left(0 \mid i c_{3}\right), g_{15}^{\prime}=\left(0 \mid i c_{3}^{2}\right), g_{19}^{\prime}=$ $=\left(\frac{\boldsymbol{a}_{1}}{2} \mid i c_{2}\right), g_{20}^{\prime}=\left(\frac{\boldsymbol{a}_{1}}{2} \mid i c_{6}^{5}\right)$ and $g_{21}^{\prime}=\left(\frac{\boldsymbol{a}_{1}}{2} \mid i c_{6}\right)$. The squares of those elements can be easily calculated: $\left(g_{4}^{\prime}\right)^{2}=(0 \mid q),\left(g_{5}^{\prime}\right)^{2}=(0 \mid q),\left(g_{6}^{\prime}\right)^{2}=(0 \mid q)$, $\left(g_{10}^{\prime}\right)^{2}=(0 \mid q), \quad\left(g_{11}^{\prime}\right)^{2}=(0 \mid q), \quad\left(g_{12}^{\prime}\right)^{2}=(0 \mid q)$, $\left(g_{13}^{\prime}\right)^{2}=(0 \mid e),\left(g_{14}^{\prime}\right)^{2}=\left(0 \mid c_{3}^{2}\right),\left(g_{15}^{\prime}\right)^{2}=\left(0 \mid q c_{3}\right)$, $\left(g_{19}^{\prime}\right)^{2}=(0 \mid q),\left(g_{20}^{\prime}\right)^{2}=\left(0 \mid q c_{3}^{2}\right)$ and $\left(g_{21}^{\prime}\right)^{2}=\left(0 \mid c_{3}\right)$.

After calculating the Herring criteria, it is easy to see that all, both single- and two-valued, irreducible projective representations at this point belong to the case $a_{2}$ [9] (subscript 2 means that $\boldsymbol{k}$ is not equivalent to $-\boldsymbol{k}$, but the space group contains an element $R$ that transforms $\boldsymbol{k}$ into $-\boldsymbol{k}$ ), when there is no additional degeneration of the states provided that their invariance with respect to the time reversal is taken into account. The consistency conditions for the irreducible projective representations along the direction $\Gamma-\Delta-A$, which is the direction of the highest symmetry in the Brillouin zone of a crystal structure with the spatial symmetry group $P 6_{3} / m m c\left(D_{6 h}^{4}\right)$, were shown in Fig. 5 of work [1].

\subsection{Line $\mathrm{K}-\mathrm{P}-\mathrm{H}$ of crystalline graphite and point $K$ of single-layer graphene}

\subsubsection{Redesignation}

First of all, let us make an important clarification. The matrices of the irreducible representations of the wave-vector groups, $D_{\boldsymbol{k}}(h)$, and their characters $\chi_{D_{\boldsymbol{k}}(h)}$ contain the phase factor $e^{-i \boldsymbol{k}(\boldsymbol{\alpha}+\boldsymbol{a})}$, where $\boldsymbol{k}$ is the wave vector, $\boldsymbol{\alpha}$ the vector of nontrivial translation, and $\boldsymbol{a}$ the vector of trivial translation for the element $g=(\boldsymbol{\alpha}+\boldsymbol{a} \mid r)$ of the spatial symmetry group. This factor was not taken into consideration, when constructing the characters of irreducible representations in work [1] (see formulas (6) and (7) in work [1]). For the basic elements of the spatial group, one can choose $\mathbf{a}=0$, so that this phase factor takes 
the form $e^{-i \mathbf{k} \boldsymbol{\alpha}}$, where the nontrivial translation vector $\boldsymbol{\alpha}$ corresponds to the "rotational" element $r$, i.e. to the basic element $h=(\boldsymbol{\alpha} \mid r)$ of the spatial symmetry group. The account for the phase factor $e^{-i \boldsymbol{k} \boldsymbol{\alpha}}$ for the elements $r$ 's is necessary, when constructing a correct irreducible projective representation of the wave-vector group in the cases where the scalar product $(\boldsymbol{k}, \boldsymbol{\alpha}) \neq 0$. Note that in the case of point $A$ considered above, the nontrivial translation vector $\boldsymbol{\alpha}$ was always equal to zero for the nonzero characters of "rotational" elements $r$ 's.

Since the phase factor $e^{-i \mathbf{k} \boldsymbol{\alpha}}(r)$ is inherent to the spatial symmetry of a periodic structure, it can be introduced into the definition of the atomic equivalence representation by replacing formula (15) in work [1] by the formula

$\chi_{\text {eq }}\left(R_{\alpha}\right)=e^{-i \boldsymbol{k} \boldsymbol{\alpha}}\left(R_{\alpha}\right) \sum_{j} \delta_{R_{\alpha} \boldsymbol{r}_{j}, \boldsymbol{r}_{j}} e^{i \boldsymbol{K}_{m} \boldsymbol{r}_{j}}$,

denoting the previously used $\chi_{\text {eq }}\left(R_{\alpha}\right)$ without the phase factor as $\left(\chi_{\text {eq }}\right)_{0}\left(R_{\alpha}\right)$, i.e. the new $D_{\text {eq }}$ will contain the phase factor $e^{-i \boldsymbol{k} \boldsymbol{\alpha}}\left(R_{\alpha}\right)$, and also denoting $D_{\text {eq }}$ obtained for various points in the Brillouin zone in work [1] and not containing the phase factor as $\left(D_{\text {eq }}\right)_{0}$.

At points $K$ and $H$ of crystalline graphite $\gamma-C$ and point $K$ of single-layer graphene $C_{L, 1}$, the factor-

Table 8. Characters of the irreducible

projective representations of the projective classes $K_{0}$ and $K_{1}$ of the group $\overline{6} m 2\left(D_{3 h}\right)$ corresponding to the standard factor-systems $\omega_{(0)}^{\prime}\left(r_{2}, r_{1}\right)$ (the unity-containing factor-system for ordinary vector representations) and $\omega_{(1)}^{\prime}\left(r_{2}, r_{1}\right)$, respectively

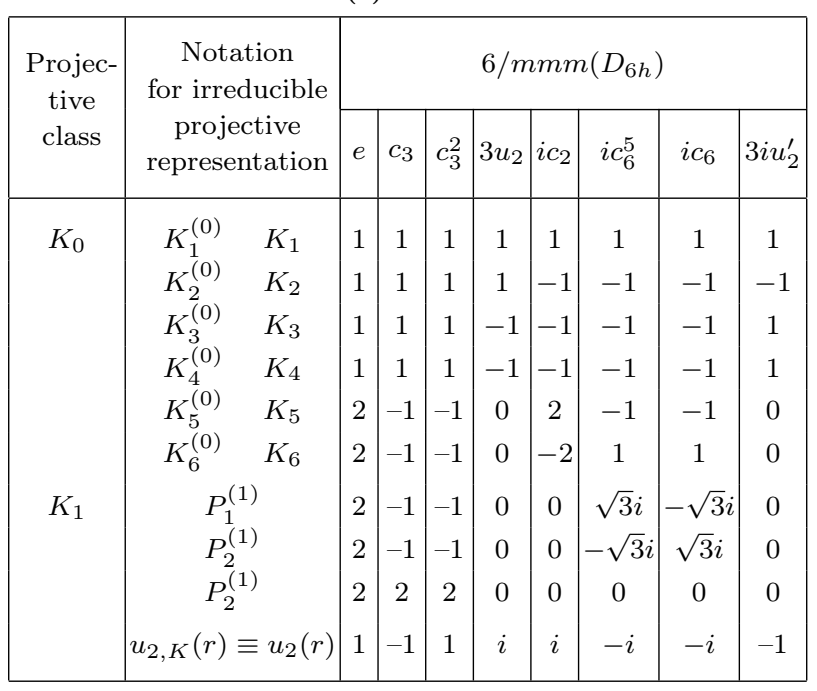

groups of wave-vector groups are isomorphic to the same point symmetry group $\overline{6} m 2\left(D_{3 h}\right)$ with respect to the invariant translational subgroups.

Each of the stars of the wave-vector groups at points $K$ in both crystalline graphite $\gamma-C$ and singlelayer graphene $C_{L, 1}$ contains two vectors. These are the vectors $\left(\boldsymbol{k}_{K}\right)_{1}=-\frac{1}{3}\left(2 \boldsymbol{b}_{2}-\boldsymbol{b}_{3}\right)$ and $\left(\boldsymbol{k}_{K}\right)_{2}=$ $=\frac{1}{3}\left(2 \boldsymbol{b}_{2}-\boldsymbol{b}_{3}\right)$ for crystalline graphite, and the vectors $\left(\boldsymbol{k}_{K}\right)_{1}=-\frac{1}{3}\left(2 \boldsymbol{b}_{1}-\boldsymbol{b}_{2}\right)$ and $\left(\boldsymbol{k}_{K}\right)_{2}=\frac{1}{3}\left(2 \boldsymbol{b}_{1}-\boldsymbol{b}_{2}\right)$ for single-layer graphene. For crystalline graphite, the nontrivial translation vector $\boldsymbol{\alpha}=\boldsymbol{a}_{1} / 2$ is perpendicular to the wave vectors $\left(\boldsymbol{k}_{K}\right)_{1}$ and $\left(\boldsymbol{k}_{K}\right)_{2}$. Therefore, the phase factor $e^{-i \boldsymbol{k}_{K} \boldsymbol{\alpha}}$ equals 1 in all cases. This is also true for single-layer graphene as well, where the nontrivial translation vector equals zero. This means that, for both structures, the factor-systems $\omega_{1, K}\left(r_{2}, r_{1}\right)$ include the " +1 "-values only, i.e. they are completely unity-containing factorsystems, which coincide with the standard factorsystems of the projective class $K_{0}$. At the same time, since $\omega_{2, K}\left(r_{2}, r_{1}\right)=\omega_{1, K}\left(r_{2}, r_{1}\right) \omega_{2}\left(r_{2}, r_{1}\right)=$ $=\omega_{(0)}^{\prime}\left(r_{2}, r_{1}\right) \omega_{2}\left(r_{2}, r_{1}\right)=\omega_{2}\left(r_{2}, r_{1}\right)$, the factor-systems for the projective representations at points $K$ taking the spin into account, i.e. $\omega_{2, K}\left(r_{2}, r_{1}\right)$, coincide with the factor-system $\omega_{2}\left(r_{2}, r_{1}\right)$ belonging to the projective class $K_{1}$.

The characters of the irreducible projective representations of the projective classes $K_{0}$ (where they coincide with the ordinary or vector representations) and $K_{1}$ of the group $\overline{6} m 2\left(D_{3 h}\right)$, which correspond to the standard factor-systems $\omega_{(0)}^{\prime}\left(r_{2}, r_{1}\right)$ (a unitycontaining factor-system, whose coefficients equal only to +1$)$ and $\omega_{(1)}^{\prime}\left(r_{2}, r_{1}\right)$, respectively, are presented in Table 8 . The characters of two-dimensional irreducible projective representations corresponding to the standard factor-system $\omega_{(1)}^{\prime}\left(r_{2}, r_{1}\right)$ are marked with the symbol $P$. The coefficients $u_{2, K}(r) \equiv u_{2}(r)$ are given in the bottom part of Table 8 .

In Table 9, the characters of the two-valued (spinor) irreducible projective representations of points $K$ in the Brillouin zones of crystalline graphite and single-layer graphene are given. They are identical for those two structures.

It should be noted that, for the two-valued (spinor) irreducible projective representations of points $K$ in the Brillouin zones of crystalline graphite and graphene, the following equations are satisfied:

$\left(K^{\prime}\right)_{i}^{(1)}=u_{2}(r) P_{i}^{(1)}$.

ISSN 2071-0194. Ukr. J. Phys. 2020. Vol. 65, No. 4 
In addition, for various points in the Brillouin zones e.g., for points $K$ - we can also determine how the characters of the representations of the direct products $K_{i} \otimes D_{1 / 2}^{+}$, where $K_{i}$ is the character of the $i$ th projective irreducible representation making no allowance for the electron spin, can be expanded in twovalued (spinor) irreducible projective representations with regard for the electron spin. In other words, we can determine which orbitals or the sum of orbitals found making allowance for the spin will correspond to an orbital obtained without regard for the spin, if the latter is taken into account.

Let us determine this correspondence proceeding from the distributions of the electron $\pi$-band representations at points $K$ in crystalline graphite and single-layer graphene without taking the electron spin into account (see Table 12 in work [1]). In particular, for the products $K_{2}^{(0)} \otimes D_{1 / 2}^{+}, K_{4}^{(0)} \otimes D_{1 / 2}^{+}$, and $K_{6}^{(0)} \otimes D_{1 / 2}^{+}$, whose characters are presented in the bottom part of Table 9 , it is easy to find the consistency conditions for the irreducible projective representations, which arise, when the electron spin is taken into account for the orbitals $K_{i}$ determined without regard for the spin. In particular, the orbital $\left(K^{\prime}\right)_{1}^{(1)}$, which makes allowance for the electron spin, corresponds to the orbital $K_{2}^{(0)}$, if the spin is taken into account; the orbital $\left(K^{\prime}\right)_{1}^{(1)}$ also corresponds to the orbital $K_{4}^{(0)}$; the sum of orbitals $\left(K^{\prime}\right)_{2}^{(1)}+\left(K^{\prime}\right)_{3}^{(1)}$ corresponds to the orbital $K_{6}^{(0)}$, i.e. if the electron spin is taken into account, the orbital $K_{6}^{(0)}$, which is doubly degenerate, if the electron spin is not taken into account, becomes split into two doubly degenerate spin orbitals $\left(K^{\prime}\right)_{2}^{(1)}$ and $\left(K^{\prime}\right)_{3}^{(1)}$.

The characters of projective representations for points $K$, namely, the equivalence representations $K_{\text {eq }}$, the representations of the spatial symmetry of $\pi$-orbitals $K_{z}$, the representations of the symmetry of electron $\pi$-bands without regard for the electron spin $K_{\pi}$, two-valued representations $D_{1 / 2}^{+}, K_{z}^{\prime}$, and representations of the symmetry of electron $\pi^{\prime}$-bands with regard for the electron spin $K_{\pi}^{\prime}$, are presented in Table 2. The distributions of electron excitations for $\pi$-bands (without taking and taking the electron spin into account) over the two-valued (spinor) irreducible projective representations of points $K$ in the Brillouin zones of crystalline graphite $\gamma-C$ and singlelayer graphene $C_{L, 1}$ are presented in Table 3 .
An important consequence of the account for the electron spin for electron excitations at points $K$ is the splitting of the doubly degenerate spinless orbitals $K_{6}^{(0)}$ in the structures of both crystalline graphite and single-layer graphene. This splitting is predicted by the theoretical-group analysis and occurs as a result of the consideration of the spin-orbit interaction. But it is extremely weak for carbon structures (about $1.0 \div 1.5 \mathrm{meV}[2]$ ) and will not be noticeable against the state energies measured in electronvolts and even tens of electronvolts. From the viewpoint of a theoretical group description, this splitting of the spinless orbitals $K_{6}^{(0)}$ into the spin orbitals $\left(K^{\prime}\right)_{2}^{(1)}$ and $\left(K^{\prime}\right)_{3}^{(1)}$, if the spin is taken into account, has a principal character for noncarbon structures with the spatial symmetry group $P 6_{3} / m m c\left(D_{6 h}^{4}\right)$ as well. For instance, it can be significant for dichalcogenides of transition metals.

\subsubsection{Point $H$}

As was mentioned above, the wave-vector factorgroup at point $H$ in the Brillouin zone of crystalline graphite $\gamma-C$ with respect to the infinite invariant translation subgroup is isomorphic to the point group $\overline{6} m 2\left(D_{3 h}\right)$. The star of the wave-vector group contains two vectors, $\left(\boldsymbol{k}_{H}\right)_{1}=-\frac{1}{2} \boldsymbol{b}_{1}-\frac{1}{3}\left(2 \boldsymbol{b}_{2}-\boldsymbol{b}_{3}\right)$ and $\left(\boldsymbol{k}_{H}\right)_{2}=-\frac{1}{2} \boldsymbol{b}_{1}+\frac{1}{3}\left(2 \boldsymbol{b}_{2}-\boldsymbol{b}_{3}\right)$.

Table 9. Characters of the two-valued (spinor) irreducible projective representations of the group $\overline{6} m 2\left(D_{3 h}\right)$ (the projective class $K_{1}$ ) of the spinor representation $D_{1 / 2}^{+}(r)$ and the projective representation products $K_{2}^{(0)} \otimes D_{1 / 2}^{+}$, $K_{4}^{(0)} \otimes D_{1 / 2}^{+}$, and $K_{6}^{(0)} \otimes D_{1 / 2}^{+}$

\begin{tabular}{|c|c|c|c|c|c|c|c|c|c|}
\hline \multirow{2}{*}{$\begin{array}{l}\text { Projec- } \\
\text { tive } \\
\text { class }\end{array}$} & \multirow{2}{*}{$\begin{array}{l}\text { Notation } \\
\text { for irreducible } \\
\text { projective } \\
\text { representation }\end{array}$} & \multicolumn{8}{|c|}{$\overline{6} m 2\left(D_{3 h}\right)$} \\
\hline & & $e$ & $c_{3}$ & $c_{3}^{2}$ & $3 u_{2}$ & $i c_{2}$ & $i c_{6}^{5}$ & $i c_{6}$ & $3 i u_{2}^{\prime}$ \\
\hline \multirow[t]{4}{*}{$K_{1}$} & $\left(K^{\prime}\right)_{1}^{(1)}$ & 2 & 1 & -1 & 0 & 0 & $\sqrt{3}$ & $-\sqrt{3}$ & 0 \\
\hline & $\left(K^{\prime}\right)_{2}^{(1)}$ & 2 & 1 & -1 & 0 & 0 & $-\sqrt{3}$ & $\sqrt{3}$ & 0 \\
\hline & $\left(K^{\prime}\right)_{3}^{(1)}$ & 2 & -2 & 2 & 0 & 0 & 0 & 0 & 0 \\
\hline & $D_{1 / 2}^{+}(r)$ & 2 & 1 & -1 & 0 & 0 & $-\sqrt{3}$ & $\sqrt{3}$ & 0 \\
\hline \multirow[t]{3}{*}{$K_{1}$} & $K_{2}^{(0)} \otimes D_{1 / 2}^{+}$ & 2 & 1 & -1 & 0 & 0 & $\sqrt{3}$ & $-\sqrt{3}$ & 0 \\
\hline & $K_{4}^{(0)} \otimes D_{1 / 2}^{+}$ & 2 & 1 & -1 & 0 & 0 & $\sqrt{3}$ & $-\sqrt{3}$ & 0 \\
\hline & $K_{6}^{(0)} \otimes D_{1 / 2}^{+}$ & 4 & -1 & 1 & 0 & 0 & $-\sqrt{3}$ & $\sqrt{3}$ & 0 \\
\hline
\end{tabular}


Table 10. Characters of $(a)$ the one-valued irreducible projective representations of the projective class $K_{1}$ of the group $\overline{6} m 2\left(D_{3 h}\right)$ corresponding to its standard factor-system and $(b)$ the one-valued $p$-equivalent representations describing the symmetry of vibrational and electron excitations without taking the spin into account at point $H$ in the Brillouin zone of crystalline graphite for the spatial symmetry group (the wave-vector group of point $H)$ that is a subgroup of the spatial symmetry group $P 6_{3} / \mathrm{mmc}\left(D_{6 h}^{4}\right)$

\begin{tabular}{|c|c|c|c|c|c|c|c|c|c|c|}
\hline \multirow{2}{*}{$\begin{array}{c}\text { Projec- } \\
\text { tive } \\
\text { class }\end{array}$} & \multirow{2}{*}{\multicolumn{2}{|c|}{$\begin{array}{l}\text { Notation } \\
\text { for irreducible } \\
\text { projective } \\
\text { representation }\end{array}$}} & \multicolumn{8}{|c|}{$\overline{6} m 2\left(D_{3 h}\right)$} \\
\hline & & & $e$ & $c_{3}$ & $c_{3}^{2}$ & $3 u_{2}$ & $i c_{2}$ & $i c_{6}^{5}$ & $i c_{6}$ & $3 i u_{2}^{\prime}$ \\
\hline \multirow[t]{6}{*}{$K_{1}$} & \multirow[t]{3}{*}{$a$} & $P_{1}^{(1)}$ & 2 & -1 & -1 & 0 & 0 & $\sqrt{3} i$ & $-\sqrt{3} i$ & 0 \\
\hline & & $P_{2}^{(1)}$ & 2 & -1 & -1 & 0 & 0 & $-\sqrt{3} i$ & $\sqrt{3} i$ & 0 \\
\hline & & $P_{3}^{(1)}$ & 2 & 2 & 2 & 0 & 0 & 0 & 0 & 0 \\
\hline & \multirow{6}{*}{$b$} & $u_{1, H}(r)$ & 1 & 1 & 1 & 1 & $i$ & $i$ & $i$ & $i$ \\
\hline & & $e^{i \boldsymbol{k}_{H} \boldsymbol{a}}(r)$ & 1 & 1 & 1 & 1 & $i$ & $i$ & $i$ & $i$ \\
\hline & & $e^{i \boldsymbol{k}_{H} \boldsymbol{a}}(r) u_{1, H}(r)$ & 1 & 1 & 1 & 1 & -1 & -1 & -1 & -1 \\
\hline \multirow[t]{3}{*}{$K_{1}$} & & $H_{1}^{(1)}$ & 2 & -1 & -1 & 0 & 0 & $-\sqrt{3} i$ & $\sqrt{3} i$ & 0 \\
\hline & & $H_{2}^{(1)}$ & 2 & -1 & -1 & 0 & 0 & $\sqrt{3} i$ & $-\sqrt{3} i$ & 0 \\
\hline & & $H_{3}^{(1)}$ & 2 & 2 & 2 & 0 & 0 & 0 & 0 & 0 \\
\hline
\end{tabular}

In work [1], it was shown that both the factorsystem of the spatial symmetry group of crystalline graphite at point $H$ of its Brillouin zone, $\omega_{1, H}\left(r_{2}, r_{1}\right)$, with the coefficients $u_{1, H}(r)$ of reduction to the standard form and the factor-system of the spin variable transformations, $\omega_{2}\left(r_{2}, r_{1}\right)$, with the coefficients $u_{2}(r)$ of reduction to the standard form belong to the same projective class $K_{1}$. This means that the two-valued (spinor) irreducible projective representations for point $H$ in the Brillouin zone of crystalline graphite $\gamma-C$ belong to the projective class $K_{0}\left(K_{1} \cdot K_{1}=K_{0}\right)$, and the reduction coefficients of the transformation factor-system to the standard form taking the electron spin into account, $\omega_{2, H}\left(r_{2}, r_{1}\right)=\omega_{1, H}\left(r_{2}, r_{1}\right) \omega_{2}\left(r_{2}, r_{1}\right)$, are determined at this point by the equality $u_{2, H}(r)=$ $=u_{1, H}(r) u_{2}(r)$. When constructing the characters of the two-valued (spinor) irreducible projective representations of point $H$, it is also necessary to consider the phase factor $e^{-i \boldsymbol{k}_{H} \boldsymbol{\alpha}}(r)$, i.e. to determine the resulting factors $e^{-i \boldsymbol{k}_{H} \boldsymbol{\alpha}}(r) u_{1, H}(r) u_{2}(r)$, by which the characters of the irreducible projective representations of the projective class $K_{0}$ (these are the characters of the vector representations for the point symmetry group $\left.\overline{6} m 2\left(D_{3 h}\right)\right)$ have to be multiplied.

The characters of the single-valued irreducible projective representations of the projective class $K_{1}$ of the point group $\overline{6} m 2\left(D_{3 h}\right)$, which correspond to the standard factor-system of this class, and the characters of the single-valued irreducible projective representations characterizing the spatial symmetry of vibrational and electron - in the latter case, not taking the electron spin into account - excitations at point $H$ in the Brillouin zone of crystalline graphite are given in Tables 10, $a$ and $10, b$, respectively ${ }^{2}$. The bottom part of Table 10, $a$ contains the values of the coefficients $u_{1, H}(r)$ that transform the factor-system of the projective class $K_{1}$, which corresponds to the spatial symmetry of point $H$, to the standard form, as well as the values of the phase factors $e^{-i \mathbf{k}_{H} \boldsymbol{\alpha}}(r)$ and the coefficient products $e^{-i \mathbf{k}_{H} \boldsymbol{\alpha}}(r) u_{1, H}(r)$.

The characters of the two-valued (spinor) irreducible projective representations belonging to the projective class $K_{0}$ and characterizing the symmetry of electron excitations taking the electron spin

2 It is of interest to note that the characters of the singlevalued irreducible projective representations for point $H$ in the Brillouin zone of crystalline graphite (the spatial symmetry group of crystalline graphite is $P 6_{3} / m m c\left(D_{6 h}^{4}\right)$, and the factor-group of the wave-vector group for point $H$ is isomorphic to the point group $\overline{6} m 2\left(D_{3 h}\right)$ with respect to the invariant translation subgroup), which belong to the projective class $K_{1}$, exactly coincide with the characters of the first three irreducible representations presented in Table C.28 of work [8].

ISSN 2071-0194. Ukr. J. Phys. 2020. Vol. 65, No. 4 
Table 11. Characters of (a) the two-valued (spinor) irreducible projective representations at point $H$ in the Brillouin zone of crystalline graphite $\gamma-C$ (the spatial symmetry group $P 6_{3} / m m c\left(D_{6 h}^{4}\right)$, the projective class $\left.K_{0}\right)$ and $(b)$ the projective representation products $H_{1}^{(1)} \otimes D_{1 / 2}^{+}$and $H_{3}^{(1)} \otimes D_{1 / 2}^{+}$

\begin{tabular}{|c|c|c|c|c|c|c|c|c|c|}
\hline \multirow{2}{*}{$\begin{array}{c}\text { Projec- } \\
\text { tive } \\
\text { class }\end{array}$} & \multirow{2}{*}{$\begin{array}{l}\text { Notation } \\
\text { for irreducible } \\
\text { projective } \\
\text { representation }\end{array}$} & \multicolumn{8}{|c|}{$6 / m m m\left(D_{6 h}\right)$} \\
\hline & & $e$ & $c_{3}$ & $c_{3}^{2}$ & $3 u_{2}$ & $i c_{2}$ & $i c_{6}^{5}$ & $i c_{6}$ & $3 i u_{2}^{\prime}$ \\
\hline \multirow[t]{11}{*}{$K_{0}$} & $a \quad\left(H^{\prime}\right)_{1}^{(0)}$ & 1 & -1 & 1 & $i$ & $-i$ & $i$ & $i$ & 1 \\
\hline & $\left(H^{\prime}\right)_{2}^{(0)}$ & 1 & -1 & 1 & $i$ & $i$ & $-i$ & $-i$ & -1 \\
\hline & $\left(H^{\prime}\right)_{3}^{(0)}$ & 1 & -1 & 1 & $-i$ & $-i$ & $i$ & $i$ & -1 \\
\hline & $\left(H^{\prime}\right)_{4}^{(0)}$ & 1 & -1 & 1 & $-i$ & $i$ & $-i$ & $-i$ & 1 \\
\hline & $\left(H^{\prime}\right)_{5}^{(0)}$ & 2 & 1 & -1 & 0 & $-2 i$ & $-i$ & $-i$ & 0 \\
\hline & $\left(H^{\prime}\right)_{6}^{(0)}$ & 2 & 1 & -1 & 0 & $2 i$ & $i$ & $i$ & 0 \\
\hline & $u_{1, H}(r)$ & 1 & 1 & 1 & 1 & $i$ & $i$ & $i$ & $i$ \\
\hline & $u_{2}(r)$ & 1 & -1 & 1 & $i$ & $i$ & $-i$ & $-i$ & -1 \\
\hline & $e^{i \boldsymbol{k}_{H} \boldsymbol{a}}(r)$ & 1 & 1 & 1 & 1 & $i$ & $i$ & $i$ & $i$ \\
\hline & $e^{i \boldsymbol{k}_{H} \boldsymbol{a}}(r) u_{1, H}(r) u_{2}(r)$ & 1 & -1 & 1 & $i$ & $-i$ & $i$ & $i$ & 1 \\
\hline & $D_{1 / 2}^{+}(r)$ & 2 & 1 & -1 & 0 & 0 & $-\sqrt{3}$ & $\sqrt{3}$ & 0 \\
\hline \multirow[t]{3}{*}{$K_{0}$} & $H_{1}^{(1)} \otimes D_{1 / 2}^{+}$ & 4 & -1 & 1 & 0 & 0 & $3 i$ & $3 i$ & 0 \\
\hline & $H_{3}^{(1)} \otimes D_{1 / 2}^{+}$ & 4 & 2 & -2 & 0 & 0 & 0 & 0 & 0 \\
\hline & $H_{\pi}^{\prime}$ & 8 & 1 & -1 & 0 & 0 & $3 i$ & $3 i$ & 0 \\
\hline
\end{tabular}

into account are given in Table 11, a. The bottom part of Table 11, a contains the values of the coefficients $u_{1, H}(r)$ and $u_{2}(r)$, which transform the factor-system $\omega_{1, H}\left(r_{2}, r_{1}\right)$ and $\omega_{2}\left(r_{2}, r_{1}\right)$, respectively, to the standard form, as well as the values of the phase factors $e^{-i \mathbf{k}_{H} \boldsymbol{\alpha}}(r)$, the coefficient products $e^{-i \mathbf{k}_{H} \boldsymbol{\alpha}}(r) u_{1, H}(r) u_{2}(r)$, and the characters of the double-digit spinor representation $D_{1 / 2}^{+}(r)$ of the point symmetry group $\overline{6} m 2\left(D_{3 h}\right)$. In Table $11, b$, the characters of the products of the projectivev representations $H_{1}^{(1)} \otimes D_{1 / 2}^{+}$and $H_{3}^{(1)} \otimes D_{1 / 2}^{+}$are shown, which make it is easy to find the consistency conditions for the irreducible projective representations for the spinless orbitals of point $H$, the orbitals $H_{i}^{(1)}$ of the projective class $K_{1}$ with irreducible projective representations of spin orbitals $\left(H^{\prime}\right)_{i}^{(0)}$ of the projective class $K_{0}$, which arise, when the electron spin is taken into account. For instance, if the electron spin is taken into consideration, the sum of spin orbitals $\left(\left(H^{\prime}\right)_{1}^{(0)}+\left(H^{\prime}\right)_{3}^{(0)}\right)+\left(H^{\prime}\right)_{6}^{(0)}$ corresponds to the spinless orbital $H_{1}^{(1)}$, and the sum of spin orbitals $\left(H^{\prime}\right)_{5}^{(0)}+\left(H^{\prime}\right)_{6}^{(0)}$ to the spinless orbital $H_{3}^{(1)}$.
The characters of the projective representation of all electron $\pi$-bands taking the electron spin into account ( $\pi^{\prime}$-bands) for point $H$ in the Brillouin zone of crystalline graphite $\gamma-C$, i.e. the representation $H_{\pi}^{\prime}$, are equal to the sums of the corresponding character values of all spin orbitals $\left(H^{\prime}\right)_{i}^{(0)}$. They are given in the bottom part of Table 11, $b$. The indicated values for the characters of the projective representation $H_{\pi}^{\prime}$ can be easily obtained using formula (4) (for point $H$, it looks like $H_{\pi}^{\prime}=H_{\text {eq }} \otimes H_{z}^{\prime}$, where $H_{z}^{\prime}$ is a representation that characterizes the symmetry of a $\pi$-electron with its spin at point $H$ in the Brillouin zone of crystalline graphite).

Table 2 demonstrates the characters of the equivalence representation for point $H$ in the Brillouin zone of crystalline graphite, the representations $H_{\text {eq }}$; the representations $H_{z} \equiv \Gamma_{z}$ and $H_{\pi}=H_{e q} \otimes H_{z}$ describing the spatial symmetry of $\pi$-electron and electron $\pi$-bands, respectively, without taking the spin into account; the two-valued (spinor) representation $D_{1 / 2}^{+}$, and the two-valued representations $H_{z}^{\prime}=H_{z} \otimes D_{1 / 2}^{+}$ and $H_{\pi}^{\prime}=H_{\text {eq }} \otimes H_{z}^{\prime}$ describing the symmetry of $\pi$ electron and electron $\pi$-bands making allowance for 
the spin. Table 3 contains the distribution of electron $\pi^{\prime}$-bands with regard for the spin at point $H$ over the two-valued (spinor) irreducible projective representations of the projective class $K_{0}$.

Our calculation of the Herring criterion for point $H$ with the use of the symmetry elements satisfying the equality $g^{\prime} \boldsymbol{k}=-\boldsymbol{k}$ - namely, these are the elements $g_{7}^{\prime}=\left(\frac{\boldsymbol{a}_{1}}{2} \mid c_{2}\right), g_{8}^{\prime}=\left(\frac{\boldsymbol{a}_{1}}{2} \mid c_{6}^{5}\right), g_{9}^{\prime}=\left(\frac{\boldsymbol{a}_{1}}{2} \mid c_{6}\right), g_{10}^{\prime}=$ $=\left(\frac{\boldsymbol{a}_{1}}{2} \mid\left(u_{2}^{\prime}\right)_{1}\right), g_{11}^{\prime}=\left(\frac{\boldsymbol{a}_{1}}{2} \mid\left(u_{2}^{\prime}\right)_{2}\right), g_{12}^{\prime} \stackrel{2}{=}\left(\frac{\boldsymbol{a}_{1}}{2} \mid\left(u_{2}^{\prime}\right)_{3}\right)$,

Table 12. (a) Characters of the one-valued irreducible projective representations of the projective class $K_{0}$ of the group $3 m\left(C_{3 v}\right)$, which describe the symmetry of vibrational and electron excitations without taking the spin into account for the spatial symmetry group at point $P$ (the wave-vector group of point $P$ ) in the Brillouin zone of crystalline graphite and are $p$-equivalent to the characters of the one-valued irreducible projective representations corresponding to the standard unity-containing factor-system of this projective class. (b) Characters of the two-valued (spinor) irreducible projective representations of point $P$ in the Brillouin zone of crystalline graphite $\gamma-C$ (the spatial symmetry group $P 6_{3} / m m c\left(D_{6 h}^{4}\right)$, the projective class $\left.K_{0}\right)$, the two-valued spinor irreducible projective representation $D_{1 / 2}^{+}$, the projective representation products $P_{1}^{(0)} \otimes D_{1 / 2}^{+}, P_{2}^{(0)} \otimes D_{1 / 2}^{+}$, and $P_{3}^{(0)} \otimes D_{1 / 2}^{+}$, and the twovalued projective representation of electron $\pi^{\prime}$-bands taking the electron spin into account - representation $P_{\pi}^{\prime}(\delta)$

\begin{tabular}{|c|c|c|c|c|c|c|}
\hline \multirow{2}{*}{$\begin{array}{l}\text { Projec- } \\
\text { tive } \\
\text { class }\end{array}$} & \multirow{2}{*}{\multicolumn{2}{|c|}{$\begin{array}{l}\text { Notation for } \\
\text { irreducible } \\
\text { projective } \\
\text { representation }\end{array}$}} & \multicolumn{4}{|c|}{$3 m\left(C_{3 v}\right)$} \\
\hline & & & $e$ & $c_{3}$ & $c_{3}^{2}$ & $3 i u_{2}^{\prime}$ \\
\hline \multirow[t]{4}{*}{$K_{0}$} & $a$ & $P_{1}^{(0)}$ & 1 & 1 & 1 & $\eta_{k_{z}}$ \\
\hline & & $P_{2}^{(0)}$ & 1 & 1 & 1 & $-\eta_{k_{z}}$ \\
\hline & & $P_{3}^{(0)}$ & 2 & -1 & -1 & 0 \\
\hline & & $u_{2}(r)$ & 1 & -1 & 1 & $i$ \\
\hline \multirow[t]{8}{*}{$K_{0}$} & $b$ & $\left(P^{\prime}\right)_{1}^{(0)}$ & 1 & -1 & 1 & $i \eta_{k_{z}}$ \\
\hline & & $\left(P^{\prime}\right)_{2}^{(0)}$ & 1 & -1 & 1 & $-i \eta_{k_{z}}$ \\
\hline & & $\left(P^{\prime}\right)_{3}^{(0)}$ & 2 & 1 & -1 & 0 \\
\hline & & $D_{1 / 2}^{+}$ & 2 & 1 & -1 & 0 \\
\hline & & $P_{1}^{(0)} \otimes D_{1 / 2}^{+}$ & 2 & 1 & -1 & 0 \\
\hline & & $P_{2}^{(0)} \otimes D_{1 / 2}^{+}$ & 2 & 1 & -1 & 0 \\
\hline & & $P_{3}^{(0)} \otimes D_{1 / 2}^{+}$ & 4 & -1 & 1 & 0 \\
\hline & & & 8 & 1 & -1 & 0 \\
\hline
\end{tabular}

${ }^{*} \eta_{k_{z}}=e^{-i \boldsymbol{k}_{P} \boldsymbol{a}_{1} / 2}=e^{-i k_{z} a_{1} / 2}$ $g_{13}^{\prime}=(0 \mid i), g_{14}^{\prime}=\left(0 \mid i c_{3}\right), g_{15}^{\prime}=\left(0 \mid i c_{3}^{2}\right), g_{16}^{\prime}=$ $=\left(0 \mid i\left(u_{2}\right)_{1}\right), g_{17}^{\prime}=\left(0 \mid i\left(u_{2}\right)_{2}\right), g_{18}^{\prime}=\left(0 \mid i\left(u_{2}\right)_{3}\right)$, and their squares equal $\left(g_{7}^{\prime}\right)^{2}=\left(\boldsymbol{a}_{1} \mid q\right),\left(g_{8}^{\prime}\right)^{2}=\left(\boldsymbol{a}_{1} \mid q c_{3}^{2}\right)$, $\left(g_{9}^{\prime}\right)^{2}=\left(\boldsymbol{a}_{1} \mid c_{3}\right),\left(g_{10}^{\prime}\right)^{2}=(0 \mid q),\left(g_{11}^{\prime}\right)^{2}=(0 \mid q)$, $\left(g_{12}^{\prime}\right)^{2}=(0 \mid q),\left(g_{13}^{\prime}\right)^{2}=(0 \mid e),\left(g_{14}^{\prime}\right)^{2}=\left(0 \mid c_{3}^{2}\right)$, $\left(g_{15}^{\prime}\right)^{2}=\left(0 \mid q c_{3}\right),\left(g_{16}^{\prime}\right)^{2}=(0 \mid q),\left(g_{17}^{\prime}\right)^{2}=(0 \mid q)$ and $\left(g_{18}^{\prime}\right)^{2}=(0 \mid q)$ - testifies that the two-valued one-dimensional spinor irreducible projective representations $\left(H^{\prime}\right)_{1}^{(0)}$ and $\left(H^{\prime}\right)_{3}^{(0)}$ at point $H$ in the Brillouin zone of crystalline graphite belong to the case $b_{2}$ [9] and, owing to their time-reversal invariance, they must unite, although our case corresponds to the union of nonequivalent complex representations, rather than complex-conjugate ones. At the same time, the two-valued two-dimensional complex-conjugate irreducible projective representations $\left(H^{\prime}\right)_{5}^{(0)}$ and $\left(H^{\prime}\right)_{6}^{(0)}$ belong to the case $a_{2}[9]$ and do not unite, if the time-reversal symmetry is taken into account.

\subsubsection{Point $P$}

The group of equivalent directions of the wave-vector group at point $P$ in the Brillouin zone of crystalline graphite $\gamma-C$ is the group $3 m\left(C_{3 v}\right)$. The group $3 m\left(C_{3 v}\right)$ has only one class of projective representations. This is the class $K_{0}$. Therefore, all projective representations of this group are $p$-equivalent to vector ones. The wave-vector star at this point contains four rays: $\left(\boldsymbol{k}_{P}\right)_{1}=-\boldsymbol{k}_{z}-\frac{1}{3}\left(2 \boldsymbol{b}_{2}-\boldsymbol{b}_{3}\right)$, $\left(\boldsymbol{k}_{P}\right)_{2}=-\boldsymbol{k}_{z}+\frac{1}{3}\left(2 \boldsymbol{b}_{2}-\boldsymbol{b}_{3}\right),\left(\boldsymbol{k}_{P}\right)_{3}=\boldsymbol{k}_{z}-\frac{1}{3}\left(2 \boldsymbol{b}_{2}-\boldsymbol{b}_{3}\right)$, and $\left(\boldsymbol{k}_{P}\right)_{4}=\boldsymbol{k}_{z}+\frac{1}{3}\left(2 \boldsymbol{b}_{2}-\boldsymbol{b}_{3}\right)$.

Table 12 presents $(a)$ the one-valued irreducible projective representations of the projective class $K_{0}$ of the group $3 m\left(C_{3 v}\right)$ at point $P$ in the Brillouin zone of crystalline graphite and (b) the two-valued (spinor) irreducible projective representations, also belonging to the projective class $K_{0}$ of this group, of equivalent directions; the characters of the spinor representation $D_{1 / 2}^{+}$; the products of projective representations $P_{1}^{(0)} \otimes D_{1 / 2}^{+}, \quad P_{2}^{(0)} \otimes D_{1 / 2}^{+}$, and $P_{3}^{(0)} \otimes D_{1 / 2}^{+}$; and the characters of the projective representation of electron $\pi^{\prime}$-bands making allowance for the electron spin, $P_{\pi}^{\prime}$. The bottom part of Table 12 , a contains the coefficients $u_{2}(r)$ that transform the factor-system of transformations of the spin variable $\omega_{2}\left(r_{2}, r_{1}\right)$ to the standard form $\omega_{2}^{\prime}\left(r_{2}, r_{1}\right) \equiv \omega_{(0)}^{\prime}\left(r_{2}, r_{1}\right.$. The bottom part of the whole Table 12 shows the phase factor value.

ISSN 2071-0194. Ukr. J. Phys. 2020. Vol. 65, No. 4 
The characters of the one-valued projective equivalence representations $P_{\text {eq }}$, the one-valued projective representations $P_{z}$ and $P_{\pi}$, the double-valued projective representations $D_{1 / 2}^{+}$and $P_{z}^{\prime}$, and the two-valued (spinor) projective representation $P_{\pi}^{\prime}$ of electron $\pi^{\prime}$ bands making allowance for the electron spin for point $P$ in the Brillouin zone of crystalline graphite are given in Table 2. The distributions of projective representations of electron $\pi$-bands without taking the electron spin into account, $P_{\pi}$, and taking it into account, $P_{\pi}^{\prime}$, over the irreducible one- and two-valued, respectively, projective representations are presented in Table 3.

The calculation of the Herring criterion for point $P$ in the Brillouin zone of crystalline graphite with the use of the symmetry elements satisfying the requirement $g^{\prime} \boldsymbol{k}=-\boldsymbol{k}$-namely, these are the elements $g_{10}^{\prime}=$ $=\left(\frac{\boldsymbol{a}_{1}}{2} \mid\left(u_{2}^{\prime}\right)_{1}\right), g_{11}^{\prime}=\left(\frac{\boldsymbol{a}_{1}}{2} \mid\left(u_{2}^{\prime}\right)_{2}\right), g_{12}^{\prime}=\left(\frac{\boldsymbol{a}_{1}}{2} \mid\left(u_{2}^{\prime}\right)_{3}\right)$, $g_{13}^{\prime}=(0 \mid i), g_{14}^{\prime}=\left(0 \mid i c_{3}\right)$ and $g_{15}^{\prime}=\left(0 \mid i c_{3}^{2}\right)$, and their squares equal $\left(g_{10}^{\prime}\right)^{2}=(0 \mid q),\left(g_{11}^{\prime}\right)^{2}=(0 \mid q)$, $\left(g_{12}^{\prime}\right)^{2}=(0 \mid q),\left(g_{13}^{\prime}\right)^{2}=(0 \mid e),\left(g_{14}^{\prime}\right)^{2}=\left(0 \mid c_{3}^{2}\right)$, $\left(g_{15}^{\prime}\right)^{2}=\left(0 \mid q c_{3}\right)$ - shows that the two-valued onedimensional spinor irreducible projective representations at this point, $\left(P^{\prime}\right)_{1}^{(0)}$ and $\left(P^{\prime}\right)_{2}^{(0)}$, belong to the case $b_{2}[9]$ and, owing to the time-reversal invariance, they unite into the two-dimensional projective representation $\left(\left(P^{\prime}\right)_{1}^{(0)}+\left(P^{\prime}\right)_{2}^{(0)}\right)$. At the same time, the one-valued irreducible projective representations $P_{1}^{(0)}, P_{2}^{(0)}, P_{3}^{(0)}$, and the two-value (spinor) projective representation $\left(P^{\prime}\right)_{3}^{(0)}$ belong to the case $a_{2}[9]$ and do not unite, if the time-reversal symmetry is taken into consideration.

At point $H$, which is limiting for points $P$ in the Brillouin zone of crystalline graphite, if the electron spin is neglected, i.e. when the phase factor $e^{-i k_{z} a_{1} / 2}=i$ at $k_{z}=-b_{1} / 2$, the sums of the characters of one-valued irreducible projective representations $P_{1}^{(0)}$ and $P_{2}^{(0)}$, as the complex-conjugate representations, should transform into the characters of the two-dimensional one-valued irreducible projective representation $H_{3}^{(1)}$ belonging to the projective class $K_{1}$ of a higher symmetry group than the symmetry group of points $P$. It is also easy to see from Table $12, b$ that, if the electron spin is taken into account, the spin orbital $\left(P^{\prime}\right)_{3}^{(0)}$ corresponds to the spinless orbitals $P_{1}^{(0)}$ and $P_{2}^{(0)}$, and the sum of the united spin orbitals $\left(\left(P^{\prime}\right)_{1}^{(0)}+\left(P^{\prime}\right)_{2}^{(0)}\right)$ and the spin orbital $\left(P^{\prime}\right)_{3}^{(0)}$ corresponds to the spinless orbital $P_{3}^{(0)}$.

ISSN 2071-0194. Ukr. J. Phys. 2020. Vol. 65, No. 4

\subsection{Line $M-U-L$ of crystalline} graphite and point $M$ of single-layer graphene

At points $M$ and $L$ in the Brillouin zone of crystalline graphite $\gamma-C$ and at point $M$ in the Brillouin zone of single-layer graphene $C_{L, 1}$, the factor-groups of wavevector groups with respect to the invariant translation subgroups are isomorphic to the same point symmetry group $m m m\left(D_{2 h}\right)$, which is the point symmetry group of equivalent directions for points $M$ in those structures.

\subsubsection{Points $M$}

Each of the stars of the wave-vector groups at points $M$ in the Brillouin zones of the structures concerned contains three rays: these are $\left(\boldsymbol{k}_{M}\right)_{1}=-\frac{1}{2} \boldsymbol{b}_{3}$, $\left(\boldsymbol{k}_{M}\right)_{2}=\frac{1}{2} \boldsymbol{b}_{2}$, and $\left(\boldsymbol{k}_{M}\right)_{3}=-\frac{1}{2}\left(\boldsymbol{b}_{2}-\boldsymbol{b}_{3}\right)$ for crystalline graphite $\gamma-C$; and $\left(\boldsymbol{k}_{M}\right)_{1}=-\frac{1}{2} \boldsymbol{b}_{2},\left(\boldsymbol{k}_{M}\right)_{2}=$ $=\frac{1}{2} \boldsymbol{b}_{1}$, and $\left(\boldsymbol{k}_{M}\right)_{3}=-\frac{1}{2}\left(\boldsymbol{b}_{1}-\boldsymbol{b}_{2}\right)$ for single-layer graphene $C_{L, 1}$.

First of all, as was done in work [1] for the point group $6 / \mathrm{mmm}\left(D_{6 h}\right)$, let us construct a factor system $\omega_{2}\left(r_{2}, r_{1}\right)$ describing the transformations of spinors under the action of symmetry operations for the point group $\mathrm{mmm}\left(D_{2 h}\right)$ and determine the coefficients $u_{2}(r)$ that transform it to the standard form $\omega_{2}^{\prime}\left(r_{2}, r_{1}\right)$.

Of three rays of the wave-vector stars at point $M$ in the Brillouin zones of both crystalline graphite and single-layer graphene, let us consider the rays $\left(\boldsymbol{k}_{M}\right)_{1}$ (points $M_{1}$ ), for which the elements of symmetry that transform these rays into the equivalent ones and form the point symmetry group $\mathrm{mmm}$ are the elements $e,\left(u_{2}\right)_{1}, c_{2},\left(u_{2}^{\prime}\right)_{1}, i, i\left(u_{2}\right)_{1}, i c_{2}$, and $i\left(u_{2}^{\prime}\right)_{1}$. As the $\mathrm{mmm}$ group generators, let us choose the elements $a=\left(u_{2}\right)_{1}, b=c_{2}$, and $c=i$. This choice makes allowance for the composition principle. According to the latter, the group $\mathrm{mmm}$ can be represented as the direct group product $222 \otimes \overline{1}(\mathrm{mmm}=222 \otimes \overline{1}$ or $D_{2 h}=D_{2} \otimes C_{i}$ ), and the group 222 as the direct group product $2^{\prime} \otimes 2\left(222=2^{\prime} \otimes 2\right.$ or $\left.D_{2}=C_{2}^{\prime} \otimes C_{2}\right)$.

By applying the defining relations, let us calculate all values of $\omega_{2}\left(r_{2}, r_{1}\right)$. It is clear that this is the defining relations for the double group $(\mathrm{mmm})^{\prime}$ that have to be taken for this purpose:

$a^{4}=e, \quad b^{4}=e, \quad c^{2}=e$, $a b=q b a, \quad a c=c a, \quad b c=c b$.

The factor-system $\omega_{2}\left(r_{2}, r_{1}\right)$ calculated for the $\mathrm{mmm}$ group following this way and using the method of 
Table 13. Factor-system $\omega_{2}\left(\boldsymbol{r}_{\mathbf{2}}, \boldsymbol{r}_{\mathbf{1}}\right)$ for the group $\boldsymbol{m m m}\left(\boldsymbol{D}_{\mathbf{2}}\right)(a)$ and the corresponding standard factor-system $\omega_{2}^{\prime}\left(r_{2}, r_{1}\right)(b)$. The bottom part of Table 13, $a$ contains the values of the function $u_{2}(r)$ that transforms the factor-system $\omega_{2}\left(r_{2}, r_{1}\right)$ to the standard form $\omega_{2}^{\prime}\left(r_{2}, r_{1}\right) \equiv \omega_{(1)}^{\prime}\left(r_{2}, r_{1}\right)$

\begin{tabular}{|rr|cccccccc|}
\hline$\omega_{2}\left(r_{2}, r_{1}\right)$ & & 1 & 2 & 3 & 4 & 5 & 6 & 7 & 8 \\
\hline & $r_{2}$ & $e$ & $\left(u_{2}\right)_{1}$ & $c_{2}$ & $\left(u_{2}^{\prime}\right)_{1}$ & $i$ & $i\left(u_{2}\right)_{1}$ & $i c_{2}$ & $i\left(u_{2}^{\prime}\right)_{1}$ \\
\hline 1 & $e$ & $1_{(1)}$ & $1_{(2)}$ & $1_{(3)}$ & $1_{(4)}$ & $1_{(5)}$ & $1_{(6)}$ & $1_{(7)}$ & $1_{(8)}$ \\
2 & $\left(u_{2}\right)_{1}$ & $1_{(2)}$ & $-1_{(1)}$ & $-1_{(4)}$ & $1_{(3)}$ & $1_{(6)}$ & $-1_{(5)}$ & $-1_{(8)}$ & $1_{(7)}$ \\
3 & $c_{2}$ & $1_{(3)}$ & $1_{(4)}$ & $-1_{(1)}$ & $-1_{(2)}$ & $1_{(7)}$ & $1_{(8)}$ & $-1_{(5)}$ & $-1_{(6)}$ \\
4 & $\left(u_{2}^{\prime}\right)_{1}$ & $1_{(4)}$ & $-1_{(3)}$ & $1_{(2)}$ & $-1_{(1)}$ & $1_{(8)}$ & $-1_{(7)}$ & $1_{(6)}$ & $-1_{(5)}$ \\
5 & $i$ & $1_{(5)}$ & $1_{(6)}$ & $1_{(7)}$ & $1_{(8)}$ & $1_{(1)}$ & $1_{(2)}$ & $1_{(3)}$ & $1_{(4)}$ \\
6 & $i\left(u_{2}\right)_{1}$ & $1_{(6)}$ & $-1_{(5)}$ & $-1_{(8)}$ & $1_{(7)}$ & $1_{(2)}$ & $-1_{(1)}$ & $-1_{(4)}$ & $1_{(3)}$ \\
7 & $i c_{2}$ & $1_{(7)}$ & $1_{(8)}$ & $-1_{(5)}$ & $-1_{(6)}$ & $1_{(3)}$ & $1_{(4)}$ & $-1_{(1)}$ & $-1_{(2)}$ \\
8 & $i\left(u_{2}^{\prime}\right)_{1}$ & $1_{(8)}$ & $-1_{(7)}$ & $1_{(6)}$ & $-1_{(5)}$ & $1_{(4)}$ & $-1_{(3)}$ & $1_{(2)}$ & $-1_{(1)}$ \\
& $u_{2}(r)$ & 1 & $i$ & $i$ & -1 & 1 & $i$ & $i$ & -1 \\
\hline
\end{tabular}

$a$

work [1] is shown in Table 13, a. This factor system belongs to the projective class $K_{1}$, because $\alpha=$ $=-1, \beta=1$, and $\gamma=1$ for it [1]. In Table 13, $a$, the subscripts near the coefficients of the factorsystem $\omega_{2}\left(r_{2}, r_{1}\right)$, which contain parenthesized numbers, compose a multiplication table for the elements of the $\mathrm{mmm}$ group (the numbers in parentheses indicate the numerical designations of the elements corresponding to the products $r_{2} r_{1}$ ).

With the help of the coefficients $u_{2}(r)$ given in the bottom part of Table 13, a, the factor system $\omega_{2}\left(r_{2}, r_{1}\right)$ is transformed into a $p$-equivalent blocksymmetric form, which corresponds to the definition of a standard factor system, i.e. the factorsystem $\omega_{2}^{\prime}\left(r_{2}, r_{1}\right)$. In so doing, the values of the coefficients $u_{2}(r)$ can be calculated using formulas (13.3), (14.18), and (14.19) of work [9]. Alternatively, they can be found, when constructing an extended group, the representation group [9], where they are determined by its one-valued irreducible representations, being additional to ordinary vector representations.

The values of the coefficients $u_{2}(r)$, which characterize the transformation of spin functions for identical elements $r$ belonging to different point groups - in our case, to point groups $\mathrm{mmm}$ and $6 / \mathrm{mmm}$, where the group $\mathrm{mmm}$ is also a subgroup of the group $6 / \mathrm{mmm}$ - expectedly turned out identical. This means that the factor-system presented in Table $13, b$ is really a standard factor-system for the projective class $K_{1}$ of the point group $m m m$, i.e. the factor-system $\omega_{2}^{\prime}\left(r_{2}, r_{1}\right) \equiv \omega_{(1)}^{\prime}\left(r_{2}, r_{1}\right)$. Solid lines in
Table 13, $b$ distinguish the contours of blocks, in which the coefficients have a value of -1 .

Table 14 displays the characters of the irreducible representations of the double group $(\mathrm{mmm})^{\prime}\left(D_{2 h}^{\prime}\right)$, the additional one-valued irreducible representations of which (additional to the ordinary vector one-valued irreducible representations of the group $\mathrm{mmm}$, which can be obtained from the representations of the group $(\mathrm{mmm})^{\prime}$ by simply excluding the element $q$ from all relations) are either two-valued projective or spinor representations of the $\mathrm{mmm}$ group. The spinor representations are denoted by the symbols $\left(E^{\prime}\right)^{+}$and $\left(E^{\prime}\right)^{-}$in the Mulliken notation or the symbols $\Gamma_{5}^{+}$ and $\Gamma_{5}^{-}$in the Koster notation, where the letter $\Gamma$ denotes not only their membership in a certain point group (in the given case, this is the group $\mathrm{mmm}$ ), but also in the coinciding group of equivalent directions of the wave-vector group of point $\boldsymbol{\Gamma}$ in crystals or periodic nanostructures. The symbols $K_{0}$ and $K_{1}$ denote the corresponding projective classes, and the notations $\left(\left(\Gamma^{\prime}\right)^{(1)}\right)^{+}$and $\left(\left(\Gamma^{\prime}\right)^{(1)}\right)^{-}$were proposed by us (here, the prime means the two-valued spinor representation, the superscript (the number in the parentheses) indicates the projective class, and the superscripts "+" and "-" mean the representation parity).

The characters of the irreducible representations of the point group $\mathrm{mmm}\left(D_{2 h}\right)$ of the projective classes $K_{0}$ (ordinary one-valued or vector) and $K_{1}$ (twovalued projective or spinor) for the standard factorsystems $\omega_{(0)}^{\prime}\left(r_{2}, r_{1}\right)$ and $\omega_{(1)}^{\prime}\left(r_{2}, r_{1}\right)$ of the point group $\mathrm{mmm}$ are presented in Table 15. The irreducible pro-

ISSN 2071-0194. Ukr. J. Phys. 2020. Vol. 65, No. 4 
Table 14. Characters of the irreducible representations of the double group $(\mathbf{m m m})^{\prime}\left(D_{2 h}^{\prime}\right)$

\begin{tabular}{|c|c|c|c|c|c|c|c|c|c|c|c|c|c|}
\hline \multicolumn{4}{|c|}{$(m m m)^{\prime}\left(D_{2 h}^{\prime}\right)$} & $e$ & $q$ & $\begin{array}{l}\left(u_{2}\right)_{1}, \\
q\left(u_{2}\right)_{1}\end{array}$ & $\begin{array}{l}c_{2}, \\
q c_{2}\end{array}$ & $\begin{array}{l}\left(u_{2}^{\prime}\right)_{1}, \\
q\left(u_{2}^{\prime}\right)_{1}\end{array}$ & $i$ & $q i$ & $\begin{array}{l}i\left(u_{2}\right)_{1}, \\
q i\left(u_{2}\right)_{1}\end{array}$ & $\begin{array}{l}i c_{2}, \\
q i c_{2}\end{array}$ & $\begin{array}{c}i\left(u_{2}^{\prime}\right)_{1} \\
q i\left(u^{\prime}\right)_{1}\end{array}$ \\
\hline \multirow[t]{8}{*}{$K_{0}$} & & $\Gamma_{1}^{+}$ & $A_{1}^{+}$ & 1 & 1 & 1 & 1 & 1 & 1 & 1 & 1 & 1 & 1 \\
\hline & & $\Gamma_{1}^{-}$ & $A_{1}^{-}$ & 1 & 1 & 1 & 1 & 1 & -1 & -1 & -1 & -1 & -1 \\
\hline & & $\Gamma_{2}^{+}$ & $A_{2}^{+}$ & 1 & 1 & 1 & -1 & -1 & 1 & 1 & 1 & -1 & -1 \\
\hline & & $\Gamma_{2}^{-}$ & $A_{2}^{-}$ & 1 & 1 & 1 & -1 & -1 & -1 & -1 & -1 & 1 & 1 \\
\hline & & $\Gamma_{3}^{+}$ & $B_{1}^{+}$ & 1 & 1 & -1 & 1 & -1 & 1 & 1 & -1 & 1 & -1 \\
\hline & & $\Gamma_{3}^{-}$ & $B_{1}^{-}$ & 1 & 1 & -1 & 1 & -1 & -1 & -1 & 1 & -1 & 1 \\
\hline & & $\Gamma_{4}^{+}$ & $B_{2}^{+}$ & 1 & 1 & -1 & -1 & 1 & 1 & 1 & -1 & -1 & 1 \\
\hline & & $\Gamma_{4}^{-}$ & $B_{2}^{-}$ & 1 & 1 & -1 & -1 & 1 & -1 & -1 & 1 & 1 & -1 \\
\hline \multirow[t]{2}{*}{$K_{1}$} & $\left(\left(\Gamma^{\prime}\right)^{(1)}\right)^{+}$ & $\Gamma_{5}^{+}$ & $\left(E^{\prime}\right)^{+}$ & 2 & -2 & 0 & 0 & 0 & 2 & -2 & 0 & 0 & 0 \\
\hline & $\left(\left(\Gamma^{\prime}\right)^{(1)}\right)^{-}$ & $\Gamma_{5}^{-}$ & $\left(E^{\prime}\right)^{-}$ & 2 & -2 & 0 & 0 & 0 & -2 & 2 & 0 & 0 & 0 \\
\hline
\end{tabular}

Table 15. Characters of the one-valued

(vector) and two-valued (spinor) irreducible projective representations of the group $\mathrm{mmm}\left(\mathrm{D}_{2 h}\right)$ corresponding to the standard factor systems $\omega_{(0)}^{\prime}\left(r_{2}, r_{1}\right)$ (the projective class $\left.K_{0}\right)$ and $\omega_{(1)}^{\prime}\left(r_{2}, r_{1}\right)$ (the projective class $K_{1}$ ), respectively

\begin{tabular}{|c|c|c|c|c|c|r|r|r|c|}
\hline \multicolumn{2}{|c|}{$m m m\left(D_{2 h}\right)$} & $e$ & $\left(u_{2}\right)_{1}$ & $c_{2}$ & $\left(u_{2}^{\prime}\right)_{1}$ & $i$ & $i\left(u_{2}\right)_{1}$ & $i c_{2}$ & $i\left(u_{2}^{\prime}\right)_{1}$ \\
\hline \multirow{3}{*}{$K_{0}$} & $A_{1}^{+}$ & 1 & 1 & 1 & 1 & 1 & 1 & 1 & 1 \\
& $A_{1}^{-}$ & 1 & 1 & 1 & 1 & -1 & -1 & -1 & -1 \\
& $A_{2}^{+}$ & 1 & 1 & -1 & -1 & 1 & 1 & -1 & -1 \\
& $A_{2}^{-}$ & 1 & 1 & -1 & -1 & -1 & -1 & 1 & 1 \\
& $B_{1}^{+}$ & 1 & -1 & 1 & -1 & 1 & -1 & 1 & -1 \\
& $B_{1}^{-}$ & 1 & -1 & 1 & -1 & -1 & 1 & -1 & 1 \\
& $B_{2}^{+}$ & 1 & -1 & -1 & 1 & 1 & -1 & -1 & 1 \\
& $B_{2}^{-}$ & 1 & -1 & -1 & 1 & -1 & 1 & 1 & -1 \\
& $\left(P_{1}^{(1)}\right)^{+}$ & 2 & 0 & 0 & 0 & 2 & 0 & 0 & 0 \\
& $\left(P^{(1)}\right)^{-}$ & 2 & 0 & 0 & 0 & -2 & 0 & 0 & 0 \\
\hline
\end{tabular}

jective representations for $M$ points in the Brillouin zones of crystalline graphite and single-layer graphene are identical to them and are shown in Table 16. In the group $\mathrm{mmm}\left(D_{2 h}\right)$, the second-order axis $\left(u_{2}\right)_{1}$ is a senior axis in the structurally distinguished senior subgroup [the axis $c_{2}$ is involved into the extension of the group consisting of the elements $e$ and $\left(u_{2}\right)_{1}$ (the group $C_{2}^{\prime}$ ) to the group $D_{2}$ ]. In other words, in the formation of the direct product of the groups $C_{2}^{\prime}\left[\left(U_{2}\right)_{1}\right]$ and $C_{2}$, the axis $\left(u_{2}\right)_{1}$ plays the role of the principal axis, according to which the symbols (numbers) of irreducible representations are determined. It is easy to see that the characters of the irreducible projective representations of the class $K_{1}$ of the group $\mathrm{mmm}$ for the standard factor-system of this class,
Table 16. Characters of the one-valued (vector) (the projective class $K_{0}$ ) and two-valued (spinor) (the projective class $K_{1}$ ) irreducible projective representations of points $M$ in the Brillouin zones of crystalline graphite and single-layer graphene

\begin{tabular}{|c|c|c|c|c|c|c|c|c|c|c|}
\hline & $m m m\left(D_{2 h}\right.$ & & $e$ & $\left(u_{2}\right)_{1}$ & $c_{2}$ & $\left(u_{2}^{\prime}\right)_{1}$ & $i$ & $i\left(u_{2}\right)_{1}$ & $i c_{2}$ & $i\left(u_{2}^{\prime}\right)_{1}$ \\
\hline$K_{0}$ & & $M_{1}^{+}$ & 1 & 1 & 1 & 1 & 1 & 1 & 1 & 1 \\
\hline & & $M_{1}^{-}$ & 1 & 1 & 1 & 1 & -1 & -1 & -1 & -1 \\
\hline & & $M_{2}^{+}$ & 1 & 1 & -1 & -1 & 1 & 1 & -1 & -1 \\
\hline & & $M_{2}^{-}$ & 1 & 1 & -1 & -1 & -1 & -1 & 1 & 1 \\
\hline & & $M_{3}^{+}$ & 1 & -1 & 1 & -1 & 1 & -1 & 1 & -1 \\
\hline & & $M_{3}^{-}$ & 1 & -1 & 1 & -1 & -1 & 1 & -1 & 1 \\
\hline & & $M_{4}^{+}$ & 1 & -1 & -1 & 1 & 1 & -1 & -1 & 1 \\
\hline & & $M_{4}^{-}$ & 1 & -1 & -1 & 1 & -1 & 1 & 1 & -1 \\
\hline$K_{1}$ & $\left(\left(M^{\prime}\right)^{(1)}\right)^{+}$ & $M_{5}^{+}$ & 2 & 0 & 0 & 0 & 2 & 0 & 0 & 0 \\
\hline & $\left(\left(M^{\prime}\right)^{(1)}\right)^{-}$ & $M_{5}^{-}$ & 2 & 0 & 0 & 0 & -2 & 0 & 0 & 0 \\
\hline
\end{tabular}

where they are denoted as $\left(P^{(1)}\right)^{+}$and $\left(P^{(1)}\right)^{-}$, on the one hand, and the characters of the irreducible projective representations of the class $K_{1}$ of the group $m m m$ for points $M$, which are denoted by the symbols $M_{5}^{+}$and $M_{5}^{-}$[or $\left(\left(M^{\prime}\right)^{(1)}\right)^{+}$and $\left.\left(\left(M^{\prime}\right)^{(1)}\right)^{-}\right]$, on the other hand, coincide with the character of the spinor irreducible representations of the double group $(\mathrm{mmm})^{\prime}$.

Table 2 exhibits the characters of the projective equivalence representation at point $M$ (the representation $M_{\mathrm{eq}}$ ), the characters of the representation $M_{z} \equiv \Gamma_{z}$, which determines the spatial symmetry of the $p_{z}$ orbital, the characters of the representation of the electron $\pi$-bands without taking the spin into account (the representation $M_{\pi}$ ), and the characters of the two-valued representations $D_{1 / 2}^{+}, M_{z}^{\prime} \equiv \Gamma_{z}^{\prime}$, 
Table 17. Characters of the irreducible projective representations of the projective class $K_{4}$ of the group $m m m\left(D_{2 h}\right)$ corresponding to the standard factor-system of this class, $\omega_{(4)}^{\prime}\left(r_{2}, r_{1}\right)(a)$, and the characters of two-valued (spinor) irreducible projective representations of point $L$ in the Brillouin zone of crystalline graphite $\gamma-C$ (b)

\begin{tabular}{|c|c|c|c|c|c|c|c|c|c|}
\hline \multirow{2}{*}{$\begin{array}{l}\text { Projective } \\
\text { class }\end{array}$} & \multirow{2}{*}{$\begin{array}{l}\text { Notation for irreducible } \\
\text { projective representation }\end{array}$} & \multicolumn{8}{|c|}{$m m m\left(D_{2 h}\right)$} \\
\hline & & $e$ & $\left(u_{2}\right)_{1}$ & $c_{2}$ & $\left(u_{2}^{\prime}\right)_{1}$ & $i$ & $i\left(u_{2}\right)_{1}$ & $i c_{2}$ & $i\left(u_{2}^{\prime}\right)_{1}$ \\
\hline \multirow[t]{7}{*}{$K_{4}$} & $P_{1}^{(4)}$ & 2 & 2 & 0 & 0 & 0 & 0 & 0 & 0 \\
\hline & $P_{2}^{(4)}$ & 2 & -2 & 0 & 0 & 0 & 0 & 0 & 0 \\
\hline & $u_{1, L}(r)$ & 1 & 1 & 1 & 1 & 1 & 1 & -1 & -1 \\
\hline & $u_{2}(r)$ & 1 & $i$ & $i$ & -1 & 1 & $i$ & $i$ & -1 \\
\hline & $e^{-i \boldsymbol{k}_{L} \boldsymbol{a}}(r)$ & 1 & 1 & $i$ & $i$ & 1 & 1 & $i$ & $i$ \\
\hline & $e^{i \boldsymbol{k}_{L} \boldsymbol{a}}(r) u_{1, L}(r) u_{2}(r)$ & 1 & $i$ & -1 & $-i$ & 1 & $i$ & 1 & $i$ \\
\hline & $D_{1 / 2}^{+}$ & 2 & 0 & 0 & 0 & 2 & 0 & 0 & 0 \\
\hline \multirow[t]{3}{*}{$K_{4}$} & $(4)(4)<L_{1}^{(4)}$ & 2 & $2 i$ & 0 & 0 & 0 & 0 & 0 & 0 \\
\hline & ${ }^{0}\left((L)_{1}+(L)_{2}\right) L_{2}^{(4)}$ & 2 & $-2 i$ & 0 & 0 & 0 & 0 & 0 & 0 \\
\hline & $L_{\pi}^{\prime}$ & 8 & 0 & 0 & 0 & 0 & 0 & 0 & 0 \\
\hline
\end{tabular}

and $M_{\pi}^{\prime}$. The distributions of electron excitations at points $M$ in the Brillouin zones of crystalline graphite $\gamma$-C and single-layer graphene $C_{L, 1}$ for $\pi$-bands without taking and taking the electron spin into account over the one- and two-valued (spinor), respectively, irreducible projective representations of points $M$ are given in Table 3.

\subsubsection{Point $L$}

As was already mentioned above, the factor-group of the wave-vector group with respect to the invariant translation subgroup at points $L$ in the Brillouin zone of crystalline graphite is also isomorphic to the point group $m m m\left(D_{2 h}\right)$. The wave-vector star of point $L$ for the graphite $\gamma-C$ structure also contains three vectors: these are $\left(\boldsymbol{k}_{L}\right)_{1}=-\frac{1}{2}\left(\boldsymbol{b}_{1}+\boldsymbol{b}_{3}\right),\left(\boldsymbol{k}_{L}\right)_{2}=$ $=-\frac{1}{2}\left(\boldsymbol{b}_{1}-\boldsymbol{b}_{2}\right)$, and $\left(\boldsymbol{k}_{L}\right)_{3}=-\frac{1}{2}\left(\boldsymbol{b}_{1}+\boldsymbol{b}_{2}-\boldsymbol{b}_{3}\right)$.

Similarly to what was done earlier for point $M$, of three rays of the wave-vector stars at point $L$ in the Brillouin zone of crystalline graphite, let us consider the ray $\left(\boldsymbol{k}_{L}\right)_{1}$, for which the symmetry elementsthat transform the rays of the wave-vector star at point $L$ into the equivalent ones and form the point symmetry group $m m m\left(D_{2 h}\right)$ are the elements $e,\left(u_{2}\right)_{1}, c_{2}$, $\left(u_{2}^{\prime}\right)_{1}, i, i\left(u_{2}\right)_{1}, i c_{2}$, and $i\left(u_{2}^{\prime}\right)_{1}$. Again, as was done for the group of point $M$, let us choose the elements $a=\left(u_{2}\right)_{1}, b=c_{2}$, and $c=i$ to be the $m m m$ group generators.
In work [1], it was demonstrated that the factorsystem $\omega_{1, L}\left(r_{2}, r_{1}\right)$ belongs to the projective class $K_{5}$ of the group $m m m\left(D_{2 h}\right)$ and can be reduced to the standard form $\omega_{1, L}^{\prime}\left(r_{2}, r_{1}\right) \equiv \omega_{(5)}^{\prime}\left(r_{2}, r_{1}\right)$ with the help of the coefficients $u_{1, L}(r)$ given in the bottom part of Table 17, $a$ in work [1]. It was also shown that the factor-system $\omega_{2}\left(r_{2}, r_{1}\right)$ of spinor transformations at the symmetry operations of the group $m m m\left(D_{2 h}\right)$, as it occurs for point $M$, belongs to the projective class $K_{1}$ and is reduced to the standard form $\omega_{2}^{\prime}\left(r_{2}, r_{1}\right) \equiv \omega_{(1)}^{\prime}\left(r_{2}, r_{1}\right)$ with the help of the coefficients $u_{2}(r)$ given in the bottom part of Table 13, $a$ of this work. This means that the factor-system at point $L$ making allowance for the spin, $\omega_{2, L}\left(r_{2}, r_{1}\right)$, is the product of the factor-system $\omega_{1, L}\left(r_{2}, r_{1}\right)$ (the projective class $K_{5}$ ), which is determined by the structure of the spatial group of crystalline graphite at point $L$ making no allowance for the spin, and the factor-system $\omega_{2}\left(r_{2}, r_{1}\right)$ (the projective class $K_{1}$ ), which describes the transformations of spinors at point $L$ (in the point symmetry group $m m m\left(D_{2 h}\right)$ ), i.e. $\omega_{2, L}\left(r_{2}, r_{1}\right)=\omega_{1, L}\left(r_{2}, r_{1}\right) \omega_{2}\left(r_{2}, r_{1}\right)$.

The standard factor-system taking the spin into account for point $L, \omega_{2, L}^{\prime}\left(r_{2}, r_{1}\right)=\omega_{(5)}^{\prime}\left(r_{2}, r_{1}\right) \times$ $\times \omega_{2}^{\prime}\left(r_{2}, r_{1}\right)=\omega_{(5)}^{\prime}\left(r_{2}, r_{1}\right) \omega_{(1)}^{\prime}\left(r_{2}, r_{1}\right)$, belongs to the projective class $K_{4}$ of the group $m m m\left(D_{2 h}\right)$ (because $\left.K_{5} \cdot K_{1}=K_{4}\right)$ and coincides with the standard factor-system of the projective class $K_{4}$ of the 


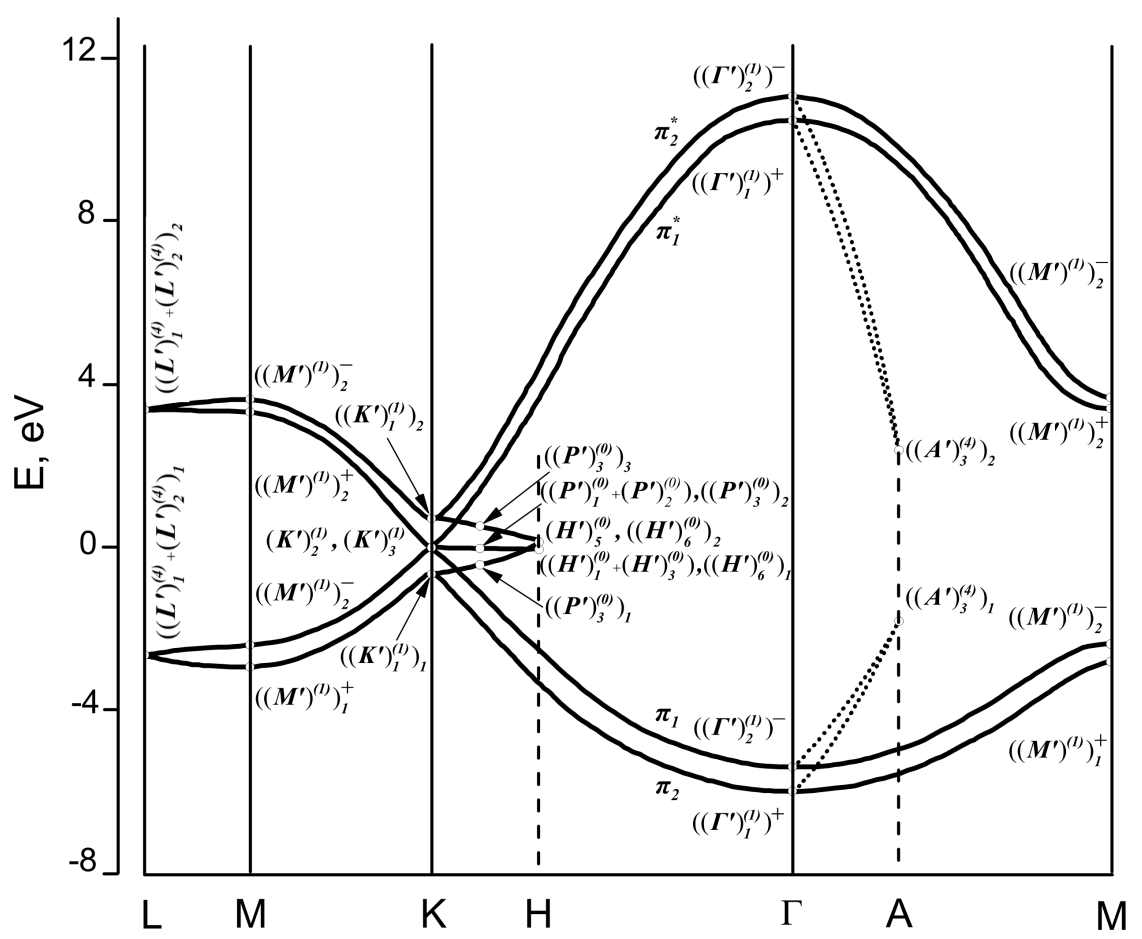

Fig. 4. Dispersion of the electron energy $\pi^{\prime}$-bands calculated taking the electron spin into account

group $m m m\left(D_{2 h}\right)$; i.e., $\omega_{2, L}^{\prime}\left(r_{2}, r_{1}\right) \equiv \omega_{(4)}^{\prime}\left(r_{2}, r_{1}\right)$ with the coefficients of transformation to the standard form, $u_{2, L}(r)$, being equal to the product of the coefficients of transformation of the factor-systems $\omega_{1, L}\left(r_{2}, r_{1}\right)$ and $\omega_{2}\left(r_{2}, r_{1}\right)$ to the standard form, $u_{1, L}(r)$ and $u_{2}(r)$.

The characters of the irreducible projective representations of the point group $\mathrm{mmm}\left(D_{2 h}\right)$ of the projective class $K_{4}$ for the standard factor-system $\omega_{(4)}^{\prime}\left(r_{2}, r_{1}\right)$ of the point group $m m m$ are presented in Table 17, $a$. In the bottom part of this table, the values of the coefficients $u_{1, L}(r), u_{2}(r)$, the phase factors $e^{-i \boldsymbol{k}_{L} \boldsymbol{\alpha}}(r)$, the products of coefficients $e^{-i \boldsymbol{k}_{L} \boldsymbol{\alpha}}(r) u_{1, L}(r) u_{2}(r) \equiv e^{-i \boldsymbol{k}_{L} \boldsymbol{\alpha}}(r) u_{2, L}(r)$, and the characters of the irreducible spinor projective representation $D_{1 / 2}^{+}$are given. The characters of the twovalued (spinor) irreducible projective representations at point $L$ in the Brillouin zone of crystalline graphite, which were calculated using the formula

$$
\begin{aligned}
& \left(L^{\prime}\right)_{i}^{(4)}=e^{-i \boldsymbol{k}_{L} \boldsymbol{\alpha}}(r)(r) u_{1, L}(r) u_{2}(r) P_{i}^{(4)}= \\
& =e^{-i \boldsymbol{k}_{L} \boldsymbol{\alpha}}(r) u_{2, L}(r) P_{i}^{(4)},
\end{aligned}
$$

are shown in Table $17, b$. The bottom part of this table contains the characters of the two-valued spinor projective representation of electron $\pi^{\prime}$-bands making allowance for the electron spin.

The characters of the projective equivalence representation at point $L$ (the representation $L_{\mathrm{eq}}$ ), the representation $L_{z} \equiv \Gamma_{z}$ determining the spatial symmetry of the $p_{z}$ orbital, the representation $L_{\pi}$ of the electron $\pi$-bands making no allowance for the spin, and the two-valued representations $D_{1 / 2}^{+}, L_{z}^{\prime} \equiv \Gamma_{z}^{\prime}$, and $L_{\pi}^{\prime}$ are given in Table 2 . The distributions of electron excitations at point $L$ in the Brillouin zone of crystalline graphite for $\pi$-bands without and with taking the electron spin into account over the one-valued and, accordingly, two-valued (spinor) irreducible projective representations of point $L$ are presented in Table 3.

Our calculation of the Herring criterion using the symmetry elements for point $L$ in the Brillouin zone of crystalline graphite, which satisfy the equality $g^{\prime} \boldsymbol{k}=-\boldsymbol{k}$ - in particular, these are the elements $g_{1}^{\prime}=(0 \mid e), g_{4}^{\prime}=\left(0 \mid\left(u_{2}\right)_{1}\right), g_{7}^{\prime}=\left(\frac{\boldsymbol{a}_{1}}{2} \mid c_{2}\right), g_{10}^{\prime}=$ $=\left(\frac{\boldsymbol{a}_{1}}{2} \mid\left(u_{2}^{\prime}\right)_{1}\right), g_{13}^{\prime}=(0 \mid i), g_{16}^{\prime}=\left(0 \mid i\left(u_{2}\right)_{1}\right), g_{19}^{\prime}=$ $=\left(\frac{\boldsymbol{a}_{1}}{2} \mid i c_{2}\right)$ and $g_{22}^{\prime}=\left(\frac{\boldsymbol{a}_{1}}{2} \mid i\left(u_{2}^{\prime}\right)_{1}\right)$, whose squares equal $\left(g_{1}^{\prime}\right)^{2}=(0 \mid e),\left(g_{4}^{\prime}\right)^{2}=(0 \mid q),\left(g_{7}^{\prime}\right)^{2}=\left(\boldsymbol{a}_{1} \mid q\right)$, $\left(g_{10}^{\prime}\right)^{2}=(0 \mid q),\left(g_{13}^{\prime}\right)^{2}=(0 \mid e),\left(g_{16}^{\prime}\right)^{2}=(0 \mid q)$, 


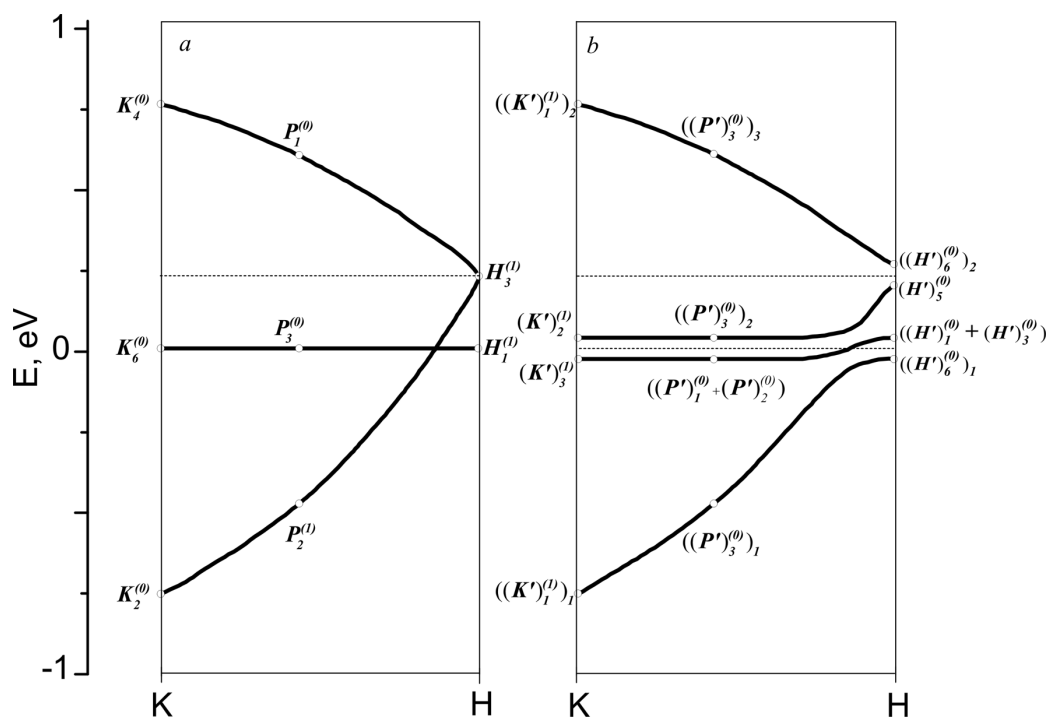

Fig. 5. Dispersion of the electron energy $(a) \pi$-bands (not taking the electron spin into account) and $(b) \pi^{\prime}-$ bands (taking the electron spin into account) in the $K-P-H$ direction of the Brillouin zone in graphite $\gamma$ - $C$ crystals. The spin-dependent fine structure of $\pi^{\prime}$-bands in panel $b$ is shown schematically, with the energy scale for the splitting of electron $\pi^{\prime}$-bands being enlarged by a factor of about $10^{3}$

$\left(g_{19}^{\prime}\right)^{2}=(0 \mid q)$ and $\left(g_{22}^{\prime}\right)^{2}=\left(\boldsymbol{a}_{1} \mid q\right),-$ testifies that the two-valued (spinor) irreducible projective representations of point $L$ in the Brillouin zone of crystalline graphite that belong to the projective class $K_{4}$ - these are the representations $\left(L^{\prime}\right)_{1}^{(4)}$ and $\left(L^{\prime}\right)_{2}^{(4)}$ - belong to the case $b_{2}$ [9]. Being the representations with complex-conjugate characters, if their time-reversal invariance is taken into consideration, they unite into four-dimensional representations $\left(\left(L^{\prime}\right)_{1}^{(4)}+\left(L^{\prime}\right)_{2}^{(4)}\right)_{1}$ and $\left(\left(L^{\prime}\right)_{1}^{(4)}+\left(L^{\prime}\right)_{2}^{(4)}\right)_{2}$, so that the degree of degeneration for each of the electron states increases to four. In Table 17, $b$, just this union of representations with complex-conjugate characters is indicated.

In Fig. 4, the dispersion of the electron energy $\pi$ bands making allowance for the electron spin $\left(\pi^{\prime}-\right.$ bands) in graphite crystals is shown schematically. The letters are used to mark points in the Brillouin zone, and the letters with indices to mark the twovalued spinor irreducible projective representations of the corresponding projective classes (the latter are indicated by the parenthesized superscripts). The dispersion of electron $\pi$-bands is schematically illustrated for all high-symmetry points in the Brillouin zone of crystalline graphite. The curves agree well at the qualitative level with the results of numerical calculations carried out in works $[11,12]$ tak- ing no electron spin into account, i.e. in the case of weak spin-orbit interaction. Nevertheless, the curves demonstrate the qualitative behavior of the dispersion of the electron $\pi$-bands along the line $\Gamma-\Delta-A$.

Figure 5, a exhibits the dispersion of the electron $\pi$-bands along the line $K-P-H$ in the Brillouin zone of crystalline graphite calculated making no allowance for the electron spin. Figure 5, b qualitatively demonstrates the spin-dependent fine structure of the energy $\pi$-bands for the splitting of electron states shown in Fig. $5, b$ (the energy scale of the spin-dependent fine structure is enlarged by a factor of about $10^{3}$ ). This fine structure is obtained, if the methods of theoretical symmetry-group analysis are consistently applied to determine the dispersion of electron $\pi$-bands in crystalline graphite taking the electron spin into account. The spin-dependent splitting can be substantial, e.g., for dichalcogenides of transition metals with the same spatial symmetry group. However, it is small for crystalline graphite and single-layer graphene, because it is caused by a low spin-orbit interaction energy for carbon atoms and, as a consequence, carbon structures.

\section{Conclusions}

1. For the first time, a theoretical symmetry-group description of the dispersion of electron $\pi$-bands

ISSN 2071-0194. Ukr. J. Phys. 2020. Vol. 65, No. 4 


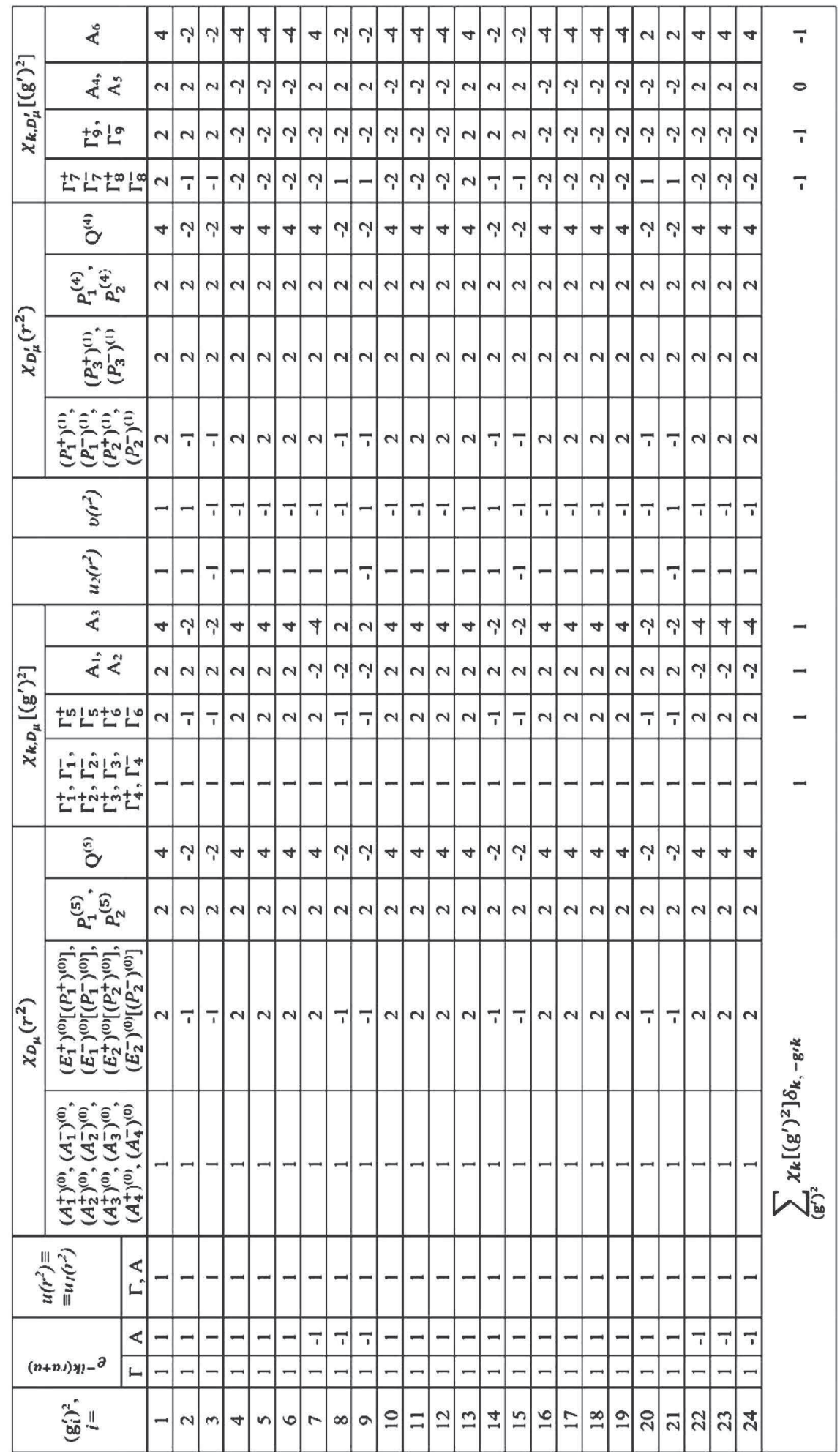


in crystalline graphite (the space symmetry group $\left.P 6_{3} / m m c\left(D_{6 h}^{4}\right)\right)$ and single-layer graphene (the diperiodic space group $P 6 / \mathrm{mmm}(D G 80)$ ) is made. The consistency conditions for the irreducible projective representations making allowance for the electron spin and the changes of the projective classes are determined for various high-symmetry points in the Brillouin zones of those materials.

2. A correlation between the electron excitations in crystalline graphite making allowance for the electron spin and the spinor excitations in single-layer graphene is shown.

3. With the help of theoretical symmetry-group methods, the existence of a fine structure for electron $\pi$-bands has been predicted for the first time. It arises, when the electron spin is taken into account, even in the case of weak spin-orbit interaction. The corresponding results include the appearance of a small (about 1.0-1.5 meV according to the estimations of work [2]) band gap between the valence and conduction bands at the Dirac points and in their very small vicinities in crystalline graphite and singlelayer graphene. A new interpretation is also given to a small splitting (the spin-dependent fine structure) of electron $\pi$-bands, if the electron spin is taken into account, at point $H$ of crystalline graphite on the basis of the established change in the projective classes of irreducible projective representations of the wavevector groups, which excludes the intersection of the dispersion curves of electron bands near point $H$.

\section{APPENDIX:}

\section{Calculation of the Herring criterion}

Table 18 illustrates the stages and the results of calculations of the characters $\chi_{\mathrm{k}, D_{\mu}}\left[\left(g^{\prime}\right)^{2}\right], \chi_{\mathrm{k}, D_{\mu}^{\prime}}\left[\left(g^{\prime}\right)^{2}\right]$, and the corresponding values of the Herring criterion for irreducible representations at points $\Gamma$ and $A$.

1. V.O. Gubanov, A.P. Naumenko, M.M. Bilyi, I.S. Dotsenko, O.M. Navozenko, M.M. Sabov, L.A. Bulavin. Energy spectra correlation of vibrational and electronic excitations and their dispersion in graphite and graphene. Ukr. J. Phys. 63, 431 (2018).

2. M.I. Katsnelson. Graphene: Carbon in Two Dimensions (Cambridge Univ. Press, 2012).

3. J.D. Bernal. The structure of graphite. Proc. Roy. Soc. London A 106, 749 (1924).

4. T. Hahn. International Tables for Crystallography. Vol. A. Space Group Symmetry (D. Reidel, 1983).
5. C. Herring. Effect on time-reversal symmetry on energy bands of crystals. Rhys. Rev. 52, 361 (1937).

6. C. Herring. Accidental degeneracy in the energy bands of crystals. Rhys. Rev. 52, 365 (1937).

7. E.A. Wood. The 80 diperiodic groups in three dimensions. Bell System Techn. J. 43, 541 (1964).

8. M.S. Dresselhaus, G. Dresselhaus, A. Jorio. Group Theory. Application to the Physics of Condensed Matter (Springer, 2008).

9. G.L. Bir, G.E. Pikus. Symmetry and Strain-Induced Effects in Semiconductors (Wiley, 1974).

10. D.S. Balchuk, M.M. Bilyi, V.P. Gryschuk, V.O. Gubanov, V.K. Kononov. Symmetry of vibrational modes, invariance of energy states to time inversion, and Raman scattering in $4 \mathrm{H}$ - and $6 \mathrm{H}-\mathrm{SiC}$ crystals. 1 . Classification of energy states in Brillouin zones. Ukr. Fiz. Zh. 41, 146 (1996) (in Ukrainian).

11. E. Doni, G. Pastori Parravicini. Energy bands and optical properties of hexagonal boron nitride and graphite. Nuovo Cimento B 64, 117 (1969).

12. F. Bassani, G. Pastori Parravicini. Electronic States and Optical Transitions in Solids (Pergamon Press, 1975).

Received 22.08.19.

Translated from Ukrainian by O.I. Voitenko

В.О. Губанов, А.П. Науменко, М.М. Білий,

І.С. Доценко, М.М. Сабов, М.С. Яхненко, Л.А. Булавін

ЕНЕРГЕТИЧНІ СПЕКТРИ ЕЛЕКТРОННИХ

ЗБУДЖЕНЬ ТА ЇХНЯ ДИСПЕРСІЯ В ГРАФІТІ

I ГРАФЕНІ: ВРАХУВАННЯ ЕЛЕКТРОННОГО

СПIНУ ТА СИМЕТРIÏ ДО IНВЕРСIї ЧАСУ

$\mathrm{P}$ е $з$ ю м е

Досліджено дисперсійні залежності електронних збуджень кристалічного графіту і одношарового графену із врахуванням спіну електрона. Визначено кореляції енергетичних спектрів електронних збуджень і, вперше, умови сумісності двозначних незвідних проективних представлень, що характеризують симетрію спінорних збуджень в зазначених вище структурах, та розподіли спінорних квантових станів за проективними класами та незвідними проективними представленнями для всіх точок високої симетрії у відповідних зонах Бріллюена. За допомогою симетрійних теоретико-групових методів для просторових груп симетрії кристалічного графіту і одношарового графену встановлено існування спін-залежних розщеплень енергетичних електронних спектрів, зокрема, розщеплень $\pi$-зон в точках Дірака, величина яких може бути значною, наприклад, для дихалькогенідів перехідних металів такої самої просторової групи симетрії, але є невеликою для кристалічного графіту і одношарового графену, оскільки вона зумовлена малою енергією спін-орбітальної взаємодії для атомів вуглецю i, як наслідок, для вуглецевих структур. 